\title{
Automorphisms of associative algebras and noncommutative geometry
}

\author{
A. Dimakis ${ }^{1}$ and F. Müller-Hoissen ${ }^{2}$ \\ ${ }^{1}$ Department of Financial and Management Engineering, University of the Aegean, \\ 31 Fostini Street, GR-82100 Chios, dimakis@aegean.gr \\ 2 Max-Planck-Institut für Strömungsforschung, Bunsenstrasse 10, D-37073 Göttingen, \\ fmuelle@gwdg.de
}

\begin{abstract}
A class of differential calculi is explored which is determined by a set of automorphisms of the underlying associative algebra. Several examples are presented. In particular, differential calculi on the quantum plane, the $h$-deformed plane and the quantum group $G L_{p, q}(2)$ are recovered in this way. Geometric structures like metrics and compatible linear connections are introduced.
\end{abstract}

\section{Introduction}

In this work $\mathcal{A}$ denotes an associative algebra, with unit $\mathbf{1}$, over a field $\mathbb{k}$ of characteristic zero (typically $\mathbb{R}$ or $\mathbb{C}$ ), and $(\Omega(\mathcal{A}), \mathrm{d})$ a differential calculus over $\mathcal{A}$. In many relevant examples in mathematics and physics (see, e.g., Ref. 1 for an introduction) the commutation relations between elements $f \in \mathcal{A}$ and 1 -forms can be expressed as

$$
\theta^{s} f=\sum_{s^{\prime} \in S} \Phi(f)_{s^{\prime}}^{s} \theta^{s^{\prime}} \quad \forall s \in S
$$

with respect to a left and right $\mathcal{A}$-module basis of $\Omega^{1}(\mathcal{A})$. Here $S$ is some finite set. Linearity and associativity then require that $f \mapsto \Phi(f)$ is an algebra isomorphism. If the basis $\left\{\theta^{s} \mid s \in S\right\}$ can be chosen in such a way that $\Phi$ is diagonal ${ }^{1}$, we obtain

$$
\theta^{s} f=\phi_{s}(f) \theta^{s}
$$

and the maps $\phi_{s}$ are automorphisms of $\mathcal{A}$.

It is not always possible to achieve the special structure (1.2). Even if it is possible, then there is in general a price to pay for it: either we have to allow for "generalized" differential calculi, or we have to extend the algebra $\mathcal{A}$ (and the differential calculus over it).

\footnotetext{
${ }^{1}$ Let us consider a change of basis $\theta^{s} \mapsto \theta^{\prime s}:=\sum_{s^{\prime} \in S} U^{s}{ }_{s^{\prime}} \theta^{s^{\prime}}$ where $U$ is an invertible matrix with entries in $\mathcal{A}$, or some extension of $\mathcal{A}$. Then (1.1) holds with the substitution $\Phi(f) \mapsto \Phi^{\prime}(f):=U \Phi(f) U^{-1}$. The problem is to find a $U$ such that $\Phi^{\prime}(f)$ is diagonal for all $f \in \mathcal{A}$.
} 
A differential calculus over $\mathcal{A}$ is an $\mathbb{N}_{0}$-graded associative algebra $\Omega(\mathcal{A})=\bigoplus_{r>0} \Omega^{r}(\mathcal{A})$ with $\Omega^{0}(\mathcal{A})=\mathcal{A}$ and $\mathcal{A}$-bimodules $\Omega^{r}(\mathcal{A})$, together with a $\mathbb{k}$-linear map d $: \Omega^{r}(\mathcal{A}) \rightarrow \Omega^{r+1}(\mathcal{A})$ satisfying $\mathrm{d}^{2}=0$ and the Leibniz rule

$$
\mathrm{d}\left(\omega \omega^{\prime}\right)=(\mathrm{d} \omega) \omega^{\prime}+(-1)^{r} \omega \mathrm{d} \omega^{\prime}
$$

for all $\omega \in \Omega^{r}(\mathcal{A})$ and $\omega^{\prime} \in \Omega(\mathcal{A})$. We require that 1 is also a unit of $\Omega(\mathcal{A})$ (which implies $\mathrm{d} \mathbf{1}=0)$. In addition one usually demands that $\Omega^{r+1}(\mathcal{A})$ coincides with the $\mathcal{A}$-bimodule generated by $\mathrm{d} \Omega^{r}(\mathcal{A})$. We shall admit, however, the possibility that the space of 1 -forms is larger than the $\mathcal{A}$-bimodule generated by $\mathrm{d} \mathcal{A}$. In this case we talk about a generalized differential calculus and denote it as $(\hat{\Omega}(\mathcal{A}), \mathrm{d})$. Of course, it always contains a sub-differential calculus $(\Omega(\mathcal{A}), \mathrm{d})$ which is an ordinary differential calculus.

There are in fact prominent examples for which the structure (1.2) appears. Consider a commutative algebra $\mathcal{A}$ with an automorphism $\phi$ and the universal differential calculus $\left(\Omega_{u}(\mathcal{A}), \mathrm{d}_{u}\right)$ over $\mathcal{A}$. Let $\left(\Omega_{\phi}(\mathcal{A}), \mathrm{d}_{\phi}\right)$ be the quotient of the universal differential graded algebra $\Omega_{u}(\mathcal{A})$ by the ideal generated by the relations $\left(\mathrm{d}_{u} a\right) b-\phi(b) \mathrm{d}_{u} a$ for all $a, b \in \mathcal{A}$. If $\phi$ is the identity, one recovers the Kähler differentials [2]. Otherwise one obtains "twisted Kähler differentials" [3]. A particular example [4, 3] is given by $\mathcal{A}=\mathbb{k}[x]$ with $\phi(f(x))=f(q x)$ for all $f \in \mathcal{A}$ and $q \in \mathbb{k} \backslash\{0\}$, so that $\mathrm{d} x f=\phi(f) \mathrm{d} x$. The structure expressed in (1.2) generalizes this construction in several ways: more than a single automorphism is admitted, the 1 -forms $\theta^{s}$ are not required to be exact, the algebra $\mathcal{A}$ need not be commutative.

Another class of examples is given by group lattice differential calculi [5, 6] which are determined by a discrete group $G$ and a finite subset $S$ not containing the unit element. The 1 -forms $\theta^{s}, s \in S$, are dual to the discrete derivatives

$$
e_{s}=\phi_{s}-\mathrm{id}
$$

acting on the algebra of functions on $G$. Here $\phi_{s}=R_{s}^{*}$ is the pull-back with the right action $R_{s}(g)=g s$ (for all $g \in G$ ). This structure generalizes as follows. Given a set of automorphisms $\phi_{s}$ of an associative algebra $\mathcal{A}$, (1.4) defines "vector fields" with which we associate, via duality relations, a set of objects $\theta^{s}$ so that (1.2) holds. It turns out that a (generalized) differential calculus can indeed be constructed in this way. An interesting aspect is that such differential calculi are naturally associated with the underlying algebra $\mathcal{A}$, since they only use properties of $\mathcal{A}$ and no "external" structures. More precisely, for this construction it is required that the automorphisms $\phi_{s}$ are sufficiently different from each other and different from the identity. On the other hand, there are examples of differential calculi over noncommutative algebras where (1.2) holds with $\phi_{s}=$ id for some or even all $s \in S$, see Ref. 7. Such calculi may be recovered as limits of curves of the kind of calculi described above (see example 4.8). But this also suggests a further generalization of the discrete derivatives (1.4) to "twisted inner derivations", see (5.1).

It turns out that the (generalized) differential calculi obtained in this way are inner at first order. Section 2 provides some information about calculi possessing this property.

Section 3 deals with homomorphisms $\mathcal{A} \rightarrow \mathcal{A}$ which are "differentiable" with respect to a differential calculus. Section 4 introduces a class of differential calculi associated with automorphisms of the underlying algebra $\mathcal{A}$ in the sense sketched above, based on the discrete derivatives (1.4). Several examples are presented. The generalization based on twisted inner 
derivations is then the subject of section 5 where in particular an example of a bicovariant differential calculus [8, 9] on the quantum group $G L_{p, q}(2)$ [10] is treated.

In section 6 we recall some facts about connections and, in particular, linear connections. Section 7 treats differential calculi associated with differentiable automorphisms in which case a (semi-) left-linear tensor product and thus close analogues of the tensors of classical differential geometry can be defined. Finally, section 8 contains some concluding remarks.

\section{Differential calculi which are inner at first order}

Let $(\hat{\Omega}(\mathcal{A}), \mathrm{d})$ be a (possibly generalized) differential calculus over $\mathcal{A}$ such that

$$
\mathrm{d} f=[\vartheta, f] \quad \forall f \in \mathcal{A}
$$

with a 1 -form $\vartheta \in \hat{\Omega}^{1}(\mathcal{A})$. In this case the first order differential calculus $\mathrm{d}: \mathcal{A} \rightarrow \hat{\Omega}^{1}(\mathcal{A})$ is said to be "inner". Of course, we may add to $\vartheta$ any 1 -form which commutes with all elements of $\mathcal{A}$. Inner differential calculi appear frequently in the literature. In particular, it seems that most bicovariant differential calculi on quantum groups have this property, see Refs. 11, $9{ }^{2}$ There are also several examples of inner first order differential calculi on quantum homogeneous spaces [12].

The expression

$$
\Delta(\omega):=[\vartheta, \omega]-\mathrm{d} \omega
$$

involving the graded commutator defines a linear map $\Delta: \hat{\Omega}(\mathcal{A}) \rightarrow \hat{\Omega}(\mathcal{A})$. As a consequence of the Leibniz rule (1.3) and the properties of the commutator, it satisfies

$$
\Delta\left(\omega \omega^{\prime}\right)=\Delta(\omega) \omega^{\prime}+(-1)^{r} \omega \Delta\left(\omega^{\prime}\right)
$$

for all $\omega \in \hat{\Omega}^{r}(\mathcal{A})$ and $\omega^{\prime} \in \hat{\Omega}(\mathcal{A})$. Hence $\Delta$ is a graded derivation of $\hat{\Omega}(\mathcal{A})$ of grade 1 . Moreover, (2.1) implies $\Delta(f)=0$ for all $f \in \mathcal{A}$, so that $\Delta$ is an $\mathcal{A}$-bimodule homomorphism, i.e.

$$
\Delta\left(f \omega f^{\prime}\right)=f \Delta(\omega) f^{\prime} \quad \forall f, f^{\prime} \in \mathcal{A}, \omega \in \hat{\Omega}(\mathcal{A})
$$

The 2-form

$$
\zeta:=\mathrm{d} \vartheta-\vartheta^{2}=\vartheta^{2}-\Delta(\vartheta)
$$

commutes with all elements of $\mathcal{A}$ :

$$
\begin{aligned}
{[\zeta, f] } & =[\mathrm{d} \vartheta, f]-\left[\vartheta^{2}, f\right]=\mathrm{d}[\vartheta, f]+[\vartheta, \mathrm{d} f]-[\vartheta,[\vartheta, f]] \\
& =\mathrm{dd} f+[\vartheta, \mathrm{d} f]-[\vartheta, \mathrm{d} f]=0
\end{aligned}
$$

Furthermore, acting with $\Delta$ on (2.2), using (2.3) and $\mathrm{d}^{2}=0$, we find

$$
\Delta^{2}(\omega)=[\Delta(\vartheta), \omega]-[\vartheta,[\vartheta, \omega]]=[\Delta(\vartheta), \omega]-\left[\vartheta^{2}, \omega\right]=-[\zeta, \omega] .
$$

An immediate consequence is the following.

\footnotetext{
${ }^{2}$ As demonstrated in Ref. 8 , the bimodule of a bicovariant first order differential calculus can always be extended with an additional generator so that the extended bimodule is also bicovariant and the differential calculus becomes inner. We then have a generalized differential calculus in the sense of the introduction.
} 
Lemma $2.1 \Delta^{2}=0$ if and only if $\zeta$ lies in the center of $\hat{\Omega}(\mathcal{A})$.

Using (2.7), we obtain

$$
\Delta(\zeta)=\Delta\left(\vartheta^{2}\right)-\Delta^{2}(\vartheta)=[\Delta(\vartheta)+\zeta, \vartheta]=\left[\vartheta^{2}, \vartheta\right]=0,
$$

so that (2.2) implies

$$
\mathrm{d} \zeta=[\vartheta, \zeta]
$$

A distinguished case occurs if $\Delta$ vanishes on $\hat{\Omega}(\mathcal{A})$. Then the differential calculus is also inner at higher orders.

\section{Differentiable maps}

A homomorphism $\phi$ of $\mathcal{A}$ is called differentiable with respect to a (possibly generalized) differential calculus $(\hat{\Omega}(\mathcal{A})$, d) if it extends to a homomorphism of $\hat{\Omega}(\mathcal{A})$ (as a graded algebra over $\mathbb{k}$ ) such that

$$
\phi \circ \mathrm{d}=\mathrm{d} \circ \phi
$$

Let $(\Omega(\mathcal{A})$, d) be the maximal ordinary differential calculus contained in $(\hat{\Omega}(\mathcal{A})$, d). Since, by use of the Leibniz rule, every element $\omega \in \Omega^{r}(\mathcal{A})$ can be expressed as

$$
\omega=\sum_{a=1}^{N} f_{(0) a} \mathrm{~d} f_{(1) a} \ldots \mathrm{d} f_{(r) a},
$$

with $f_{(p) a} \in \mathcal{A}$, we may think of extending a homomorphism $\phi$ of $\mathcal{A}$ simply by defining

$$
\phi(\omega):=\sum_{a=1}^{N} \phi\left(f_{(0) a}\right) \mathrm{d} \phi\left(f_{(1) a}\right) \ldots \mathrm{d} \phi\left(f_{(r) a}\right) .
$$

At least, this is consistent if the differential calculus is the universal one. If not, then there are special linear combinations of forms which vanish identically. But the right hand side of the above definition need not respect this and would then lead to a contradiction.

In this work, we restrict our considerations to differential calculi which are generated by first-order differential calculi without imposing further relations by hand ${ }^{3}$ on the level of $r$-forms with $r>1$. The differentiability condition for a homomorphism $\phi$ of $\mathcal{A}$ with respect to $(\Omega(\mathcal{A}), \mathrm{d})$ then becomes

$$
\sum_{a=1}^{N} f_{a} \mathrm{~d} f_{a}^{\prime}=0 \quad \Longrightarrow \quad \sum_{a=1}^{N} \phi\left(f_{a}\right) \mathrm{d} \phi\left(f_{a}^{\prime}\right)=0
$$

\footnotetext{
${ }^{3}$ An example for such a further relation would be the Woronowicz wedge product 8 which is stronger, in general, than what is required to extend a (bicovariant) first order differential calculus to higher orders.
} 
for all $f_{a}, f_{a}^{\prime} \in \mathcal{A}$ and all $N \in \mathbb{N}$. In the case of a generalized differential calculus $(\hat{\Omega}(\mathcal{A}), \mathrm{d})$, there are further relations which $\phi$ has to preserve in order to be differentiable.

Most examples of generalized differential calculi considered in this work (see section 44) are actually of a rather special kind. They are obtained by minimal extensions of $\Omega^{1}(\mathcal{A})$ in the following way. We say that an element $\alpha \in \hat{\Omega}^{1}(\mathcal{A}) \backslash \Omega^{1}(\mathcal{A})$ minimally extends $\Omega^{1}(\mathcal{A})$ if there is an $f \in \mathcal{A} \backslash\{0\}$ such that $f \alpha \in \Omega^{1}(\mathcal{A})$ and $f$ has no inverse in $\mathcal{A}$. Then $f \alpha=\sum_{a} f_{a} \mathrm{~d} f_{a}^{\prime}$ with $f_{a}, f_{a}^{\prime} \in \mathcal{A}$. In order to extend $\phi$ to $(\hat{\Omega}(\mathcal{A}), \mathrm{d})$ and thus to achieve that $\phi$ is differentiable with respect to the generalized differential calculus, we have to define $\phi(\alpha)$ in accordance with $\phi(f) \phi(\alpha)=\sum_{a} \phi\left(f_{a}\right) \mathrm{d} \phi\left(f_{a}^{\prime}\right)$.

A first order differential calculus d : $\mathcal{A} \rightarrow \hat{\Omega}^{1}(\mathcal{A})$ is called simple if there is no (nonvanishing) 1-form which commutes with all elements of $\mathcal{A}$.

Lemma 3.1 Let $\mathrm{d}: \mathcal{A} \rightarrow \hat{\Omega}^{1}(\mathcal{A})$ be inner and simple, and $\phi$ a differentiable automorphism of $\mathcal{A}$. Then

$$
\begin{aligned}
\phi(\vartheta) & =\vartheta, \\
\phi \circ \Delta & =\Delta \circ \phi .
\end{aligned}
$$

Proof: Using (2.1) we obtain $[\phi(\vartheta), \phi(f)]=\phi(\mathrm{d} f)=\mathrm{d} \phi(f)=[\vartheta, \phi(f)]$ for all $f \in \mathcal{A}$ which implies that $\phi(\vartheta)-\vartheta$ is a 1 -form which commutes with all $f \in \mathcal{A}$. But we assumed that the first order differential calculus is simple so that such a 1-form does not exist. Hence $\phi(\vartheta)-\vartheta=0$. Furthermore, applying $\phi$ to (2.2) leads to $\phi(\Delta(\omega))=[\phi(\vartheta), \phi(\omega)]-\mathrm{d} \phi(\omega)=$ $[\vartheta, \phi(\omega)]-\mathrm{d} \phi(\omega)=\Delta(\phi(\omega))$ for all $\omega \in \hat{\Omega}(\mathcal{A})$.

Remark. If the first order differential calculus is not simple, the Lemma can be generalized as follows. Let $\mathcal{Z}\left(\hat{\Omega}^{1}(\mathcal{A}), \mathcal{A}\right)$ denote the set of 1 -forms which commute with all elements of $\mathcal{A}$. Then

$$
\phi(\vartheta)=\vartheta+\vartheta_{\phi}, \quad \phi \circ \Delta(\omega)=\Delta \circ \phi(\omega)+\left[\vartheta_{\phi}, \phi(\omega)\right],
$$

with some $\vartheta_{\phi} \in \mathcal{Z}\left(\hat{\Omega}^{1}(\mathcal{A}), \mathcal{A}\right)$, depending on $\phi$.

\section{A class of differential calculi determined by auto- morphisms}

Let $G$ be a group and $S \subset G$ a finite subset which acts faithfully by automorphisms $\left\{\phi_{s} \mid s \in\right.$ $S\}$ on an associative algebra $\mathcal{A}$, so that $\phi_{s}(f)=\phi_{s^{\prime}}(f)$ for all $f \in \mathcal{A}$ implies $s=s^{\prime}$. We define maps $e_{s}: \mathcal{A} \rightarrow \mathcal{A}$ such that

$$
e_{s} f=\left[\phi_{s}(f)-f\right] / t_{s} \quad(s \in S)
$$

with an arbitrary parameter $t_{s} \in \mathbb{k}^{4}{ }^{4}$ As a consequence, the operators $e_{s}$ satisfy a twisted derivation rule:

$$
e_{s}\left(f f^{\prime}\right)=\left(e_{s} f\right) \phi_{s}\left(f^{\prime}\right)+f\left(e_{s} f^{\prime}\right) .
$$

\footnotetext{
${ }^{4}$ If $\phi_{s}$ depends on the parameter $t_{s}$ and if a norm is defined on $\mathcal{A}$, it may be of interest to consider the limit $t_{s} \rightarrow 0$. Otherwise we may set $t_{s}=1$ without restriction of generality.
} 
The linear span of mappings $\left\{e_{s} \cdot f \mid s \in S, f \in \mathcal{A}\right\}$, where

$$
\left(e_{s} \cdot f\right) f^{\prime}=\left(e_{s} f^{\prime}\right) f,
$$

is turned into an $\mathcal{A}$-bimodule $\mathcal{X}$ via

$$
f \cdot e_{s}=e_{s} \cdot \phi_{s}(f) .
$$

The elements of $\mathcal{X}$ will be called vector fields. In the following, we assume that $\mathcal{X}$ is free with $\left\{e_{s} \mid s \in S\right\}$ as a left and right $\mathcal{A}$-module basis.

Remark. Let us consider the algebra $\mathcal{A}$ obtained from $\mathbb{C}[x]$, the algebra of polynomials in a variable $x$ with coefficients in $\mathbb{C}$, by imposing the relation $x^{2}=0$. Then $\phi_{s}(x)=\mu_{s} x$, $s=1,2$, where $\mu_{s} \in \mathbb{C} \backslash\{0,1\}$ are such that $\mu_{1}^{k} \neq \mu_{2}^{l}$ for all $k, l \in \mathbb{Z} \backslash\{0\}$, determines a faithful action of $\mathbb{Z}^{2}$ by automorphisms. $e_{1} \cdot f_{1}+e_{2} \cdot f_{2}=0$ is satisfied with $f_{2}=-f_{1}\left(\mu_{1}-1\right) /\left(\mu_{2}-1\right)$ and arbitrary $f_{1} \in \mathbb{C}$. Hence $\left\{e_{s} \mid s=1,2\right\}$ is not a right $\mathcal{A}$-module basis. This example shows that a faithful action does not, in general, guarantee that $\left\{e_{s}\right\}$ is a left and right $\mathcal{A}$-module basis. In many interesting examples, the relations in $\mathcal{A}$ are much less restrictive, however, and the latter property usually holds.

Let $\hat{\Omega}^{1}(\mathcal{A})$ be the dual $\mathcal{A}$-bimodule obtained with a contraction which has the following properties:

$$
\begin{aligned}
\langle\alpha f, X\rangle & =\langle\alpha, f \cdot X\rangle \\
\langle\alpha, X \cdot f\rangle & =\langle\alpha, X\rangle f \\
\langle f \alpha, X\rangle & =f\langle\alpha, X\rangle
\end{aligned}
$$

for all $f \in \mathcal{A}, X \in \mathcal{X}$ and $\alpha \in \hat{\Omega}^{1}(\mathcal{A})$. Now

$$
\left\langle\theta^{s}, e_{s^{\prime}}\right\rangle=\delta_{s^{\prime}}^{s}
$$

defines duals of $e_{s}, s \in S$. Using (4.5) and (4.4) we obtain

$$
\begin{aligned}
\left\langle\theta^{s} f, e_{s^{\prime}}\right\rangle & =\left\langle\theta^{s}, f \cdot e_{s^{\prime}}\right\rangle=\left\langle\theta^{s}, e_{s^{\prime}} \cdot \phi_{s^{\prime}}(f)\right\rangle=\left\langle\theta^{s}, e_{s^{\prime}}\right\rangle \phi_{s^{\prime}}(f)=\delta_{s^{\prime}}^{s} \phi_{s^{\prime}}(f) \\
& =\left\langle\phi_{s}(f) \theta^{s}, e_{s^{\prime}}\right\rangle
\end{aligned}
$$

so that

$$
\theta^{s} f=\phi_{s}(f) \theta^{s} .
$$

Next we define a linear map $\mathrm{d}: \mathcal{A} \rightarrow \hat{\Omega}^{1}(\mathcal{A})$ by

$$
X f=\langle\mathrm{d} f, X\rangle
$$

for all $X \in \mathcal{X}$ and $f \in \mathcal{A}$. This implies

$$
\mathrm{d} f=\sum_{s \in S}\left(e_{s} f\right) \theta^{s} .
$$

As a consequence of (4.2) and (4.10), d satisfies the Leibniz rule

$$
\mathrm{d}\left(f f^{\prime}\right)=(\mathrm{d} f) f^{\prime}+f\left(\mathrm{~d} f^{\prime}\right)
$$


so that $\left(\hat{\Omega}^{1}(\mathcal{A}), \mathrm{d}\right)$ constitutes a (possibly generalized) first order differential calculus.

In general, it is not possible to express $\theta^{s}$ as a linear combination of differentials of elements of $\mathcal{A}$ with coefficients in $\mathcal{A}$, as in (3.2), i.e., $\theta^{s}$ need not be an element of $\Omega^{1}(\mathcal{A})$.

If there are elements $f^{s} \in \mathcal{A}, s \in S$, such that $e_{s} f^{s^{\prime}}$ is an invertible matrix and the inverse has entries in $\mathcal{A}$, then we can solve (4.12) for the $\theta^{s}$ and they lie in $\Omega^{1}(\mathcal{A})$ (see example 4.1). In this case, the elements $f^{s}$ of $\mathcal{A}$ may be regarded as coordinates.

Lemma 4.1 A 1-form $\alpha=\sum_{s \in S} \alpha_{s} \theta^{s}$ with $\alpha_{s} \in \mathcal{A}$ commutes with all elements of $\mathcal{A}$ iff

$$
\alpha_{s} \phi_{s}(f)=f \alpha_{s} \quad \forall f \in \mathcal{A}, \quad \forall s \in S .
$$

Furthermore, if $\phi_{s}(f)=U_{s} f U_{s}^{-1}$ with an invertible $U_{s} \in \mathcal{A}$, so that $\phi_{s}$ is an inner automorphism, then $\alpha_{s} U_{s}$ belongs to the center of $\mathcal{A}$.

Proof: The first statement is a direct consequence of (4.10). The second immediately follows from the first, using the special form of $\phi_{s}$.

The Lemma gives a criterion for the first order differential calculus $\mathrm{d}: \mathcal{A} \rightarrow \hat{\Omega}^{1}(\mathcal{A})$ to be simple.

Using (4.1), (4.10) and (4.12), (2.1) holds with

$$
\vartheta=\sum_{s \in S} \frac{1}{t_{s}} \theta^{s}
$$

so that all formulas of section 2 apply in the case under consideration.

Differentiability of a homomorphism $\phi: \mathcal{A} \rightarrow \mathcal{A}$ with respect to $(\Omega(\mathcal{A}), \mathrm{d})$ means that the criterion (3.4) has to be fulfilled. Differentiability of $\phi$ with respect to a generalized differential calculus $(\hat{\Omega}(\mathcal{A}), \mathrm{d})$ requires in addition that it must be possible to define $\phi$ on $\theta^{s}$ consistently in such a way that (4.12) is preserved, i.e., $\mathrm{d} \phi(f)=\sum_{s \in S} \phi\left(e_{s} f\right) \phi\left(\theta^{s}\right)$ for all $f \in \mathcal{A}$. Furthermore, (4.10) has to be preserved which leads to

$$
\phi\left(\theta^{s}\right) f=\phi \circ \phi_{s} \circ \phi^{-1}(f) \phi\left(\theta^{s}\right) .
$$

If $\phi_{s}$ is differentiable with respect to $(\hat{\Omega}(\mathcal{A}), \mathrm{d})$, then

$$
\phi_{s}\left(\theta^{s^{\prime}}\right) f=\phi_{s s^{\prime} s^{-1}}(f) \phi_{s}\left(\theta^{s^{\prime}}\right)
$$

for all $s^{\prime} \in S$ and all $f \in \mathcal{A}$. If $\operatorname{ad}(s) S \subset S$, then $\phi_{s}\left(\theta^{s^{\prime}}\right)$ satisfies the same commutation relations as $\theta^{s s^{\prime} s^{-1}}$ with elements of $\mathcal{A}$. We may then expect generically that $\phi_{s}\left(\theta^{s^{\prime}}\right)$ coincides with $\theta^{s s^{\prime} s^{-1}}$ up to a factor which lies in $\mathcal{Z}(\mathcal{A})$, the center of $\mathcal{A}$. A corresponding criterion is given next.

Lemma $4.2 A$ 1-form $\alpha=\sum_{s^{\prime} \in S} \alpha_{s^{\prime}} \theta^{s^{\prime}}$ satisfies $\alpha f=\phi_{s}(f) \alpha$ for some fixed $s \in S$ and all $f \in \mathcal{A}$ iff $\alpha_{s} \in \mathcal{Z}(\mathcal{A})$ and $\alpha_{s^{\prime}} \phi_{s^{\prime}}(f)=\phi_{s}(f) \alpha_{s^{\prime}}$ for all $s^{\prime} \in S \backslash\{s\}$ and all $f \in \mathcal{A}$. 
Proof: This is an immediate consequence of (4.10).

An element $c \in \mathcal{A}$ with the property $\phi_{s}(c)=c$ for all $s \in S$ is a constant of the differential calculus: $\mathrm{d} c=0$. The ideal $\mathcal{J}_{c}$ of $\hat{\Omega}(\mathcal{A})$, generated by $c-\lambda \mathbf{1}$ with $\lambda \in \mathbb{k}$, is a differential ideal, and $\left(\hat{\Omega}(\mathcal{A}) / \mathcal{J}_{c}, \mathrm{~d}\right)$ is again a differential calculus, but with less constants than the one we started with.

Every associative algebra admits a maximal first order differential calculus, the universal first order differential calculus $\mathrm{d}_{u}: \mathcal{A} \rightarrow \Omega_{u}^{1}(\mathcal{A})$. For each first order differential calculus $\mathrm{d}: \mathcal{A} \rightarrow \Omega^{1}(\mathcal{A})$ over $\mathcal{A}$ there is an $\mathcal{A}$-bimodule homomorphism $\pi_{1}: \Omega_{u}^{1}(\mathcal{A}) \rightarrow \Omega^{1}(\mathcal{A})$ such that $\mathrm{d}=\pi_{1} \circ \mathrm{d}_{u}$. The universal first order differential calculus extends to the universal differential calculus $\left(\Omega_{u}(\mathcal{A}), \mathrm{d}_{u}\right)$. Let $\mathcal{J}$ be the differential ideal of $\Omega_{u}(\mathcal{A})$ generated by ker $\pi_{1}$ and let

$$
\pi: \Omega_{u}(\mathcal{A}) \rightarrow \Omega(\mathcal{A}):=\Omega_{u}(\mathcal{A}) / \mathcal{J}
$$

be the corresponding projection. We define $\mathrm{d}$ on $\Omega(\mathcal{A})$ such that $\mathrm{d} \circ \pi=\pi \circ \mathrm{d}_{u}$. Since $\mathcal{J}$ is graded, $\pi$ is a graded homomorphism of the corresponding graded algebras. Then $(\Omega(\mathcal{A}), \mathrm{d})$ extends the first order differential calculus $\mathrm{d}: \mathcal{A} \rightarrow \Omega^{1}(\mathcal{A})$ to higher orders.

A generalized differential calculus is not a quotient of the universal differential calculus. In this case a corresponding extension is somewhat more subtle. But such problems are in some cases easily circumvented by appending $\mathcal{A}$ (perhaps after abstraction from a concrete realization) with suitable inverses of some of its elements such that the generalized differential calculus becomes an ordinary one over the extended algebra $\hat{\mathcal{A}}$. If a resulting formula, though obtained via manipulations with inverses, does not explicitly refer to inverses, it can be consistently restricted to the original algebra $\mathcal{A}$. There are certainly other examples which are not so easily treated.

\subsection{The structure of 2-forms and beyond}

Let us introduce the 2-forms

$$
\zeta^{g}:=\sum_{s, s^{\prime} \in S} \delta_{s s^{\prime}}^{g} \frac{1}{t_{s} t_{s^{\prime}}} \theta^{s} \theta^{s^{\prime}} \quad(g \in G) .
$$

As a consequence of (4.10), they satisfy

$$
\zeta^{g} f=\phi_{g}(f) \zeta^{g} \quad \forall f \in \mathcal{A} .
$$

In particular, $\zeta^{e}$ commutes with all $f \in \mathcal{A}$.

In order to further analyze the structure of 2-forms, we take a closer look at equation (2.5). In expanded form, using (4.15), it becomes

$$
\sum_{s, s^{\prime} \in S} \frac{1}{t_{s} t_{s^{\prime}}} \theta^{s} \theta^{s^{\prime}}=\sum_{s \in S} \frac{1}{t_{s}} \Delta\left(\theta^{s}\right)+\zeta .
$$

Multiplying from the right with $f \in \mathcal{A}$ and using the commutation relations (4.10) leads to

$$
\sum_{s, s^{\prime} \in S} \frac{1}{t_{s} t_{s^{\prime}}} \phi_{s s^{\prime}}(f) \theta^{s} \theta^{s^{\prime}}=\sum_{s \in S} \frac{1}{t_{s}} \phi_{s}(f) \Delta\left(\theta^{s}\right)+f \zeta .
$$


Since this equation holds for all $f \in \mathcal{A}$, and since we assumed that $\left\{\phi_{s} \mid s \in S\right\}$ acts faithfully on $\mathcal{A}$, we should expect that it implies

$$
\begin{aligned}
\zeta & =\sum_{s, s^{\prime} \in S} \frac{1}{t_{s} t_{s^{\prime}}} \delta_{s s^{\prime}}^{e} \theta^{s} \theta^{s^{\prime}}=\zeta^{e} \\
\Delta\left(\theta^{s}\right) & =\sum_{s^{\prime}, s^{\prime \prime} \in S} \frac{t_{s}}{t_{s^{\prime}} t_{s^{\prime \prime}}} \delta_{s^{\prime} s^{\prime \prime}}^{s} \theta^{s^{\prime}} \theta^{s^{\prime \prime}}
\end{aligned}
$$

where $e$ denotes the unit element of the group, and the 2-form relations

$$
\zeta^{g}=\sum_{s, s^{\prime} \in S} \delta_{s s^{\prime}}^{g} \frac{1}{t_{s} t_{s^{\prime}}} \theta^{s} \theta^{s^{\prime}}=0 \quad \forall g \in G \backslash(S \cup\{e\}) .
$$

Even if these relations cannot be deduced from (4.22), it is completely consistent to impose them and we will do so. A particular consequence is that the map $\Delta$ is now fixed and thus also the action of $\mathrm{d}$ on $\hat{\Omega}(\mathcal{A})$ according to $(2.2)$ :

$$
\mathrm{d} \omega=[\vartheta, \omega]-\Delta(\omega) .
$$

The step from (4.22) to (4.23)-(4.25) is a decomposition of a (general) 2-form into parts corresponding to biangles, triangles and "quadrangles" 5 :

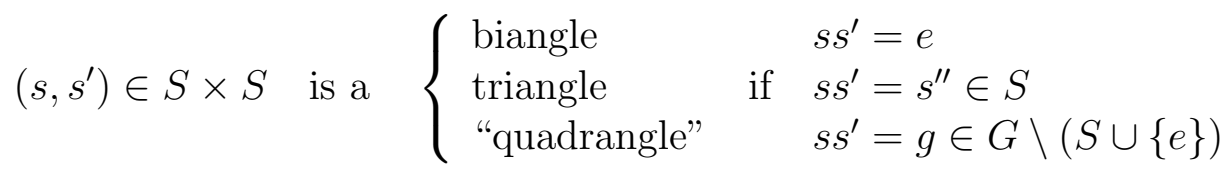

Clearly, similar decompositions can be achieved for $r$-forms with $r>2$. For a biangle, the 2-form $\theta^{s} \theta^{s^{-1}}$ commutes with all $f \in \mathcal{A}$.

\subsection{A collection of examples}

Example 4.1. The group $\mathbb{Z}$, or even the larger additive group of complex numbers, acts faithfully on $\mathcal{A}=\mathbb{C}[x]$ via automorphisms $\left(\phi_{i} f\right)(x)=f(x+i), i \in \mathbb{C}$. Let $S=\left\{i_{1}, i_{2}, \ldots, i_{n}\right\} \subset \mathbb{C}$. Then $\left\{e_{s}=\phi_{s}-\mathrm{id} \mid s \in S\right\}$ is a left and right $\mathcal{A}$-module basis of the space $\mathcal{X}$. We obtain

$$
\mathrm{d} x^{r}=\left[\left(x+i_{1}\right)^{r}-x^{r}\right] \theta^{i_{1}}+\ldots+\left[\left(x+i_{n}\right)^{r}-x^{r}\right] \theta^{i_{n}}
$$

for $r \in \mathbb{N}$. Commuting $x$ from right to left through the expression on the right hand side, using (4.10), is consistent with the left hand side because of the Leibniz rule formula $\left(\mathrm{d} x^{r}\right) x=\mathrm{d} x^{r+1}-x^{r} \mathrm{~d} x$. Next we show that the $\theta^{s}$ can be expressed in terms of differentials. (4.28) implies

$$
\left(\begin{array}{l}
\mathrm{d} x \\
\mathrm{~d} x^{2} \\
\vdots \\
\mathrm{d} x^{n}
\end{array}\right)=\left(\begin{array}{cccc}
i_{1} & i_{2} & \ldots & i_{n} \\
\left(x+i_{1}\right)^{2}-x^{2} & \left(x+i_{2}\right)^{2}-x^{2} & \ldots & \left(x+i_{n}\right)^{2}-x^{2} \\
\vdots & \vdots & & \vdots \\
\left(x+i_{1}\right)^{n}-x^{n} & \left(x+i_{2}\right)^{n}-x^{n} & \ldots & \left(x+i_{n}\right)^{n}-x^{n}
\end{array}\right)\left(\begin{array}{l}
\theta^{i_{1}} \\
\theta^{i_{2}} \\
\vdots \\
\theta^{i_{n}}
\end{array}\right)
$$

\footnotetext{
${ }^{5}$ This nomenclature is taken from Ref. $[5$. More precisely, here the notion "quadrangle" comprises all the structures different from biangles and triangles. If $\operatorname{ad}(S) S \subset S$, then these are indeed quadrangles in the sense of Ref. [5]
} 
Let $M_{S}$ denote the matrix which appears on the right hand side. Subtracting $x$ times the $k$ th row from the $(k+1)$ th row of $M_{S}$, we obtain $\operatorname{det} M_{S}=i_{1} i_{2} \cdots i_{n} \mathcal{D}\left[i_{1}, \ldots, i_{n}\right]$ with the determinant

$$
\begin{gathered}
\mathcal{D}\left[i_{1}, \ldots, i_{n}\right]=\mid \begin{array}{cccc}
1 & 1 & \cdots & 1 \\
x+i_{1} & x+i_{2} & \cdots & x+i_{n} \\
\left(x+i_{1}\right)^{2} & \left(x+i_{2}\right)^{2} & \cdots & \left(x+i_{n}\right)^{2} \\
\vdots & \vdots & \ddots & \vdots \\
\left(x+i_{1}\right)^{n-1} & \left(x+i_{2}\right)^{n-1} & \cdots & \left(x+i_{n}\right)^{n-1}
\end{array} \\
=\left|\begin{array}{cccc}
i_{3}-i_{1} & \ldots & i_{n}-i_{1} \\
\left(x+i_{2}\right)^{2}-\left(x+i_{1}\right)^{2} & \left(x+i_{3}\right)^{2}-\left(x+i_{1}\right)^{2} & \cdots & \left(x+i_{n}\right)^{2}-\left(x+i_{1}\right)^{2} \\
\vdots & \vdots & \ddots & \vdots \\
\left(x+i_{2}\right)^{n-1}-\left(x+i_{1}\right)^{n-1} & \left(x+i_{3}\right)^{n-1}-\left(x+i_{1}\right)^{n-1} & \cdots & \left(x+i_{n}\right)^{n-1}-\left(x+i_{1}\right)^{n-1}
\end{array}\right|
\end{gathered}
$$

This is the determinant of a matrix with the same structure as $M_{S}$. We can thus proceed as above and subtract $\left(x+i_{1}\right)$ times the $k$ th row from the $(k+1)$ th row. This leads to

$$
\mathcal{D}\left[i_{1}, \ldots, i_{n}\right]=\left(i_{2}-i_{1}\right)\left(i_{3}-i_{1}\right) \cdots\left(i_{n}-i_{1}\right) \mathcal{D}\left[i_{2}, \ldots, i_{n}\right]
$$

and thus

$$
\operatorname{det} M_{S}=i_{1} \cdots i_{n}\left(i_{2}-i_{1}\right) \cdots\left(i_{n}-i_{1}\right)\left(i_{3}-i_{2}\right) \cdots\left(i_{n}-i_{2}\right) \cdots\left(i_{n}-i_{n-1}\right) .
$$

In particular, $\operatorname{det} M_{S}$ does not vanish ${ }^{6}$ and does not depend on $x . M_{S}$ is therefore invertible and the 1-forms $\theta^{s}$ can be expressed as linear combinations of the differentials $\mathrm{d} x^{1}, \ldots, \mathrm{d} x^{n}$, with coefficients in $\mathcal{A}$. The automorphisms $\phi_{s}$ are differentiable with $\phi_{s}\left(\theta^{s^{\prime}}\right)=\theta^{s^{\prime}}$. Indeed, using the binomial formula, we see that the expressions (4.28) are preserved by $\phi_{s}$.

For $S=\{1\}$ we have a special case of Karoubi's construction [3] (see also Ref. 13). For $S=\{1,2\}$ we obtain

$$
\theta^{1}=2(1+x) \mathrm{d} x-\mathrm{d} x^{2}, \quad \theta^{2}=-\left(\frac{1}{2}+x\right) \mathrm{d} x+\frac{1}{2} \mathrm{~d} x^{2} .
$$

Then (4.24) leads to $\Delta\left(\theta^{1}\right)=0$ and $\Delta\left(\theta^{2}\right)=\left(\theta^{1}\right)^{2}$. From (4.25) we deduce $\theta^{2} \theta^{1}=-\theta^{1} \theta^{2}$ and $\left(\theta^{2}\right)^{2}=0$. Since there is no biangle, according to (4.23) the 2 -form $\zeta$ vanishes.

Choosing $S=\{-1,1\}$ we obtain

$$
\theta^{-1}=-\frac{1}{2}\left[(x+1)^{2}-x^{2}\right] \mathrm{d} x+\frac{1}{2} \mathrm{~d} x^{2}, \quad \theta^{1}=\frac{1}{2}\left[(x-1)^{2}-x^{2}\right] \mathrm{d} x+\frac{1}{2} \mathrm{~d} x^{2},
$$

so that $\vartheta=\mathrm{d} x^{2}-2 x \mathrm{~d} x=[\mathrm{d} x, x]$. Now (4.25) implies $\left(\theta^{-1}\right)^{2}=0=\left(\theta^{1}\right)^{2}$. There is no triangle, so (4.24) reads $\Delta=0$. But now there is a biangle and (4.23) becomes $\zeta=\theta^{-1} \theta^{1}+\theta^{1} \theta^{-1}$. Realizing $\mathcal{A}$ as the algebra of functions on $\mathbb{Z}$, we recover the one-dimensional case of the "symmetric lattice" treated in Ref. 14.

Corresponding differential calculi on $\mathbb{C}\left[x_{1}, \ldots, x_{m}\right]$ can be built as skew tensor products of such calculi.

\footnotetext{
${ }^{6}$ The $i_{k}$ are assumed to be mutually different, of course.
} 
Example 4.2. Let $\mathcal{A}$ be the algebra of $\mathbb{C}$-valued functions on a discrete group $G$. Then $G$ acts on itself by right translations which induce automorphisms $R_{g}^{*}$ of $\mathcal{A}$ via $\left(R_{g}^{*} f\right)\left(g^{\prime}\right)=f\left(g^{\prime} g\right)$. Clearly, $\left\{R_{g}^{*} \mid g \in G\right\}$ acts faithfully on $\mathcal{A}$. Let $S$ be a finite subset of $G \backslash\{e\}$. Then $(G, S)$ is called a group lattice. According to the procedure of section 4 , the set of automorphisms $\left\{R_{s}^{*} \mid s \in S\right\}$ determines a differential calculus over $\mathcal{A}$ such that $\hat{\Omega}^{1}(\mathcal{A})=\Omega^{1}(\mathcal{A})$. It is not in general inner at higher than first order. Furthermore, in general, the maps $R_{s}^{*}$ do not extend to automorphisms of the corresponding differential calculus. They do if $\operatorname{ad}(S) S \subset S$ [5]. Every first-order group lattice differential calculus is simple. Noncommutative geometry of group lattices has been elaborated in Refs. 5, 6, to which we refer the reader for further details.

Example 4.3. Let $\mathcal{A}$ be the algebra of $\mathbb{C}$-valued functions on the space $G / H$ of right $H$-cosets of a group $G$ with respect to a subgroup $H$. Then $G$ acts on $G / H$ by right translations which induce automorphisms $R_{g}^{*}$ of $\mathcal{A}$ via $\left(R_{g}^{*} f\right)\left(H g^{\prime}\right)=f\left(H g^{\prime} g\right)$. Let $S$ be a finite subset of $G \backslash\{e\}$. If certain conditions are satisfied, the set of automorphisms $\left\{R_{s}^{*} \mid s \in S\right\}$ determines a differential calculus over $\mathcal{A}$, following the procedure of section 4 . Clearly, with the choice $H=\{e\}$ we are back to our preceding example. On the other hand, if $H=G$, then $G / H$ consists of a single element only. In this case we have $R_{g}^{*}=i d$ for all $g \in G$ and therefore not a faithful action. If $H$ is a true subgroup of $G$ and if we can find a subgroup $G^{\prime}$ of $G$ such that $R_{g^{\prime}}^{*} \neq R_{g^{\prime \prime}}^{*}$ on $\mathcal{A}$ for all $g^{\prime}, g^{\prime \prime} \in G^{\prime}$ with $g^{\prime} \neq g^{\prime \prime}$, then a differential calculus can be built with each subset $S \subset G^{\prime} \backslash\{e\}$. In Ref. $[5$ we also considered coset differential calculi for which some of the $e_{s}$ vanish identically on $\mathcal{A}$. In the present work, such cases are excluded.

Example 4.4. The quantum plane is the $\mathbb{C}$-algebra $\mathcal{A}$ generated by elements $x, y$, subject to the relation $x y=q y x$ where $q \in \mathbb{C}$. An element $f(x, y) \in \mathcal{A}$ is a polynomial in the variables $x, y$. If $q \notin\{ \pm 1\}$, the only automorphisms of $\mathcal{A}$ are given by scalings of $x$ and $y$ [15]. Let us choose $S=\{\hat{1}, \hat{2}\} \subset \mathbb{Z}^{2}$, where $\hat{1}=(1,0), \hat{2}=(0,1)$, and corresponding automorphisms

$$
\left(\phi_{\hat{1}} f\right)(x, y)=f\left(\alpha^{-1} x, \beta^{-1} y\right), \quad\left(\phi_{\hat{2}} f\right)(x, y)=f\left(\gamma^{-1} x, \delta^{-1} y\right),
$$

with complex numbers $\alpha, \beta, \gamma, \delta \neq 0$. This extends to a faithful action of the group $\mathbb{Z}^{2}$ (with addition as the group composition) if $\ln \alpha \ln \delta \neq \ln \beta \ln \gamma$ (choosing an appropriate branch of the logarithm) and $(\alpha, \beta) \neq(1,1) \neq(\gamma, \delta) .\left\{e_{s} \mid s \in S\right\}$, and then also $\left\{\theta^{s} \mid s \in S\right\}$, is a left and right $\mathcal{A}$-module basis. (4.12) with (4.1), using (4.10), leads to

$$
\mathrm{d} x=A \theta^{\hat{1}} x+C \theta^{\hat{2}} x, \quad \mathrm{~d} y=B \theta^{\hat{1}} y+D \theta^{\hat{2}} y
$$

where

$$
A=(1-\alpha) / t_{\hat{1}}, \quad B=(1-\beta) / t_{\hat{1}}, \quad C=(1-\gamma) / t_{\hat{2}}, \quad D=(1-\delta) / t_{\hat{2}} .
$$

Now we obtain

$$
\begin{array}{ll}
x \mathrm{~d} x=\left(\alpha A \theta^{\hat{1}}+\gamma C \theta^{\hat{2}}\right) x^{2}, & y \mathrm{~d} x=q^{-1}\left(\beta A \theta^{\hat{1}}+\delta C \theta^{\hat{2}}\right) x y, \\
y \mathrm{~d} y=\left(\beta B \theta^{\hat{1}}+\delta D \theta^{\hat{2}}\right) y^{2}, & x \mathrm{~d} y=q\left(\alpha B \theta^{\hat{1}}+\gamma D \theta^{\hat{2}}\right) y x .
\end{array}
$$

Under what conditions on the constants appearing in these equations can we express the right hand sides in terms of $x, y, \mathrm{~d} x, \mathrm{~d} y$ ? Let us extend the algebra $\mathcal{A}$ with additional 
generators $x^{-1}, y^{-1}$ subject to the relations $x x^{-1}=\mathbf{1}=x^{-1} x$ and $y y^{-1}=\mathbf{1}=y^{-1} y$ with unit 1. The resulting algebra $\hat{\mathcal{A}}$ is known as that of the quantum torus and the maps (4.34) extend to it as automorphisms in an obvious way. Assuming $A D-B C \neq 0$, we find

$$
\theta^{\hat{1}}=(A D-B C)^{-1}\left(D \mathrm{~d} x x^{-1}-C \mathrm{~d} y y^{-1}\right), \theta^{\hat{2}}=(A D-B C)^{-1}\left(A \mathrm{~d} y y^{-1}-B \mathrm{~d} x x^{-1}\right),
$$

and the above commutation relations take the form

$$
\begin{aligned}
x \mathrm{~d} x & =(A D-B C)^{-1}\left[(\alpha A D-\gamma B C) \mathrm{d} x+(\gamma-\alpha) A C \mathrm{~d} y y^{-1} x\right] x, \\
y \mathrm{~d} x & =(A D-B C)^{-1}\left[q^{-1}(\beta A D-\delta B C) \mathrm{d} x y+(\delta-\beta) A C \mathrm{~d} y x\right], \\
y \mathrm{~d} y & =(A D-B C)^{-1}\left[(\beta-\delta) B D \mathrm{~d} x x^{-1} y+(\delta A D-\beta B C) \mathrm{d} y\right] y, \\
x \mathrm{~d} y & =(A D-B C)^{-1}[(\alpha-\gamma) B D \mathrm{~d} x y+q(\gamma A D-\alpha B C) \mathrm{d} y x] .
\end{aligned}
$$

They reduce to relations on the quantum plane if the coefficients of all terms containing $x^{-1}$ or $y^{-1}$ vanish, i.e., $(\gamma-\alpha) A C=0=(\beta-\delta) B D$. This leads to the following three cases.

a) $\delta=\beta$. Then $A C=0$, so that $A=0$ or $C=0$, which means $\alpha=1$ or $\gamma=1$. Let us choose $\gamma=1$. Then

$$
\begin{array}{ll}
x \mathrm{~d} x=\alpha \mathrm{d} x x, & y \mathrm{~d} x=q^{-1} \beta \mathrm{d} x y \\
y \mathrm{~d} y=\beta \mathrm{d} y y, & x \mathrm{~d} y=q \mathrm{~d} y x+(\beta-1) \mathrm{d} x y .
\end{array}
$$

The alternative case $\alpha=1$ leads to the same relations, but with $\alpha$ replaced by $\gamma$. With the choice $\alpha=\beta=p q$ we obtain from (4.40) the relations

$$
\begin{aligned}
& x \mathrm{~d} x=p q \mathrm{~d} x x, \quad y \mathrm{~d} x=p \mathrm{~d} x y \\
& y \mathrm{~d} y=p q \mathrm{~d} y y, \quad x \mathrm{~d} y=q \mathrm{~d} y x+(p q-1) \mathrm{d} x y .
\end{aligned}
$$

This calculus is covariant under the quantum group $G L_{p, q}(2)$ [16] (see also Ref. 17).

b) $\gamma=\alpha$. This implies $B D=0$. The two solutions which emerge in this case are mapped to (4.40) via $x \leftrightarrow y, q \mapsto q^{-1}, \alpha \mapsto \beta$, and $\delta \mapsto \alpha$, respectively $\beta \mapsto \alpha$.

c) $\gamma \neq \alpha$ and $\delta \neq \beta$. Then $A C=0=B D$, so that either $B=C=0$ or $A=D=0$. Let us consider the first case, where $\beta=\gamma=1$. Then

$$
x \mathrm{~d} x=\alpha \mathrm{d} x x, \quad y \mathrm{~d} x=q^{-1} \mathrm{~d} x y, \quad y \mathrm{~d} y=\delta \mathrm{d} y y, \quad x \mathrm{~d} y=q \mathrm{~d} y x .
$$

If $A=D=0$, so that $\alpha=\delta=1$, we obtain the same relations, but with $\alpha$ and $\delta$ replaced by $\gamma$ and $\beta$, respectively.

In the case under consideration, there are only quadrangles so that $\Delta\left(\theta^{s}\right)=0=\zeta$ and the associated 2-form relations (4.25) are

$$
\left(\theta^{\hat{1}}\right)^{2}=\left(\theta^{\hat{2}}\right)^{2}=0, \quad \theta^{\hat{1}} \theta^{\hat{2}}+\theta^{\hat{2}} \theta^{\hat{1}}=0 .
$$

As a consequence, $\mathrm{d} \vartheta=[\vartheta, \vartheta]=2 \vartheta^{2}=0$. The whole differential calculus is inner: $\mathrm{d} \omega=$ $[\vartheta, \omega]$ for all $\omega \in \hat{\Omega}(\mathcal{A})$. The first order calculus is simple if $q$ is not a root of $\beta, \delta$, and $q^{-1}$ not a root of $\alpha, \gamma$. Furthermore, using the criterion (3.4), it follows that the automorphisms $\phi_{s}$ and their inverses are differentiable with respect to each of the above differential calculi over 
the extended algebra $\hat{\mathcal{A}}$. Application to (4.35) leads to $\phi_{s}\left(\theta^{\hat{1}}\right) x=\theta^{\hat{1}} x$ and $\phi_{s}\left(\theta^{\hat{2}}\right) y=\theta^{\hat{2}} y$. In the extended algebra, this is

$$
\phi_{s}\left(\theta^{s^{\prime}}\right)=\theta^{s^{\prime}} .
$$

It follows that, with these relations, the maps $\phi_{s}$ are also differentiable with respect to each of the above (generalized) differential calculi over $\mathcal{A}$.

Example 4.5. The Heisenberg algebra is generated by two elements $x, y$, subject to the relation $[x, y]=h \mathbf{1}$ with $h \in \mathbb{C}$. Then

$$
\left(\phi_{\hat{1}} f\right)(x, y)=f(x+a \mathbf{1}, y), \quad\left(\phi_{\hat{2}} f\right)(x, y)=f(x, y+b \mathbf{1}),
$$

are automorphisms, where $a, b \in \mathbb{C} \backslash\{0\}$. This yields a faithful action of $G=\mathbb{Z}^{2}$. Choosing $t_{\hat{1}}=a$ and $t_{\hat{2}}=b$, we obtain

$$
\mathrm{d} x=\theta^{\hat{1}}, \quad \mathrm{~d} y=\theta^{\hat{2}},
$$

and the commutation relations

$$
[\mathrm{d} x, x]=a \mathrm{~d} x, \quad[\mathrm{~d} x, y]=0, \quad[\mathrm{~d} y, x]=0, \quad[\mathrm{~d} y, y]=b \mathrm{~d} y .
$$

The automorphisms $\phi_{s}$ preserve these relations and are therefore differentiable. They satisfy $\phi_{s}\left(\theta^{s^{\prime}}\right)=\theta^{s^{\prime}}$. In the limit $h \rightarrow 0$ we obtain a familiar differential calculus which supplies $\mathbb{R}^{2}$ $\left(\right.$ or $\left.\mathbb{Z}^{2}\right)$ with a quadratic lattice digraph structure [13].

Example 4.6. The algebra $\mathcal{A}$ of the $h$-deformed plane is generated by elements $x, y$, with the relation

$$
[x, y]=h y^{2}
$$

where $h \in \mathbb{C}$. Extending $\mathcal{A}$ with an element $y^{-1}$, imposing the relations $y y^{-1}=\mathbf{1}=$ $y^{-1} y$, (4.48) becomes equivalent to the correspondingly extended Heisenberg algebra with $\left[y^{-1}, x\right]=h \mathbf{1}$. Two automorphisms of the (extended) $h$-plane are given by

$$
\left(\phi_{1} f\right)(x, y)=f(x+p y, y), \quad\left(\phi_{2} f\right)(x, y)=f\left(r^{-1} x, r^{-1} y\right)
$$

with $p, r \in \mathbb{C} \backslash\{0\}$. They generate a faithful action of $\mathbb{Z}^{2}$ on $\mathcal{A}$. The 1 -forms $\theta^{\hat{1}}, \theta^{\hat{2}}$ of the associated differential calculus then satisfy

$$
f(x, y) \theta^{\hat{1}}=\theta^{\hat{1}} f(x-p y, y), \quad f(x, y) \theta^{\hat{2}}=\theta^{\hat{2}} f(r x, r y) .
$$

The vector fields given by (4.1) act on $f \in \mathcal{A}$ as follows,

$$
\left(e_{1} f\right)(x, y)=[f(x+p y, y)-f(x, y)] / t_{1}, \quad\left(e_{2} f\right)(x, y)=\left[f\left(r^{-1} x, r^{-1} y\right)-f(x, y)\right] / t_{2},
$$

and (4.12) yields

$$
\mathrm{d} x=\frac{p}{t_{1}} \theta^{\hat{1}} y+\frac{1-r}{t_{2}} \theta^{\hat{2}} x, \quad \mathrm{~d} y=\frac{1-r}{t_{2}} \theta^{\hat{2}} y .
$$


Now we obtain

$$
\begin{aligned}
& x \mathrm{~d} x=\frac{p}{t_{1}} \theta^{\hat{1}}\left[y x+(h-p) y^{2}\right]+\frac{r-r^{2}}{t_{2}} \theta^{\hat{2}} x^{2}, \quad x \mathrm{~d} y=\frac{r-r^{2}}{t_{2}} \theta^{\hat{2}}\left(y x+h y^{2}\right), \\
& y \mathrm{~d} x=\frac{p}{t_{1}} \theta^{\hat{1}} y^{2}+\frac{r-r^{2}}{t_{2}} \theta^{\hat{2}}\left(x y-h y^{2}\right), \quad y \mathrm{~d} y=\frac{r-r^{2}}{t_{2}} \theta^{\hat{2}} y^{2} .
\end{aligned}
$$

In order to replace the $\theta^{s}$ by expressions involving $\mathrm{d} x$ and $\mathrm{d} y$, we return to the extended algebra. Then

$$
\theta^{\hat{1}}=\frac{t_{1}}{p}\left(\mathrm{~d} x-\mathrm{d} y y^{-1} x\right) y^{-1}, \quad \theta^{\hat{2}}=\frac{t_{2}}{1-r} \mathrm{~d} y y^{-1}
$$

Substitution into the above commutation relations leads to

$$
\begin{aligned}
{[x, \mathrm{~d} x] } & =h^{\prime}(\mathrm{d} y(x+h y)-\mathrm{d} x y)+(r-1) \mathrm{d} y y^{-1} x^{2}, \\
{[y, \mathrm{~d} x] } & =-h \mathrm{~d} y y+(r-1) \mathrm{d} y x, \\
{[y, \mathrm{~d} y] } & =(r-1) \mathrm{d} y y \\
{[x, \mathrm{~d} y] } & =r h \mathrm{~d} y y+(r-1) \mathrm{d} y x
\end{aligned}
$$

where we introduced $h^{\prime}=p-h$. The only way to get rid of the terms containing $y^{-1}$ is to take the limit $r \rightarrow 1$. Then we recover a differential calculus which is covariant under the quantum group $G L_{h, h^{\prime}}(2)$ [8]. In this limit $\theta^{\hat{2}}$ commutes with all $f \in \mathcal{A}$. In particular, the resulting (first order) differential calculus is not simple. In order for $e_{2}$ to have a limit, we should choose $t_{2}=1-r$. $e_{2}$ then tends to an outer derivation on $\mathcal{A}$ as $r \rightarrow 1$.

From the properties of the group $\mathbb{Z}^{2}$, we deduce $\Delta\left(\theta^{s}\right)=\zeta=0$ and

$$
\left(\theta^{\hat{1}}\right)^{2}=\left(\theta^{\hat{2}}\right)^{2}=0, \quad \theta^{\hat{1}} \theta^{\hat{2}}+\theta^{\hat{2}} \theta^{\hat{1}}=0 .
$$

From these relations we obtain, in the limit $r \rightarrow 1$,

$$
(\mathrm{d} x)^{2}=h^{\prime} \mathrm{d} x \mathrm{~d} y, \quad(\mathrm{~d} y)^{2}=0, \quad \mathrm{~d} x \mathrm{~d} y+\mathrm{d} y \mathrm{~d} x=0
$$

(see also Ref. [18).

Differentiability of the maps $\phi_{s}$ (with respect to the differential calculus over the extended algebra) requires $\phi_{s}\left(\theta^{s^{\prime}}\right)=\theta^{s^{\prime}}$ via (4.54). It follows that $\phi_{s}$ preserves the relations (4.53) and is therefore indeed differentiable. $\phi_{s}$ is then also differentiable with respect to the (generalized) differential calculus over $\mathcal{A}$ obtained in the limit $r \rightarrow 1$.

Example 4.7. Let $\mathcal{A}$ be the complex algebra freely generated by $x, y, x^{-1}, y^{-1}$, subject to $x x^{-1}=\mathbf{1}=x^{-1} x$ and $y y^{-1}=\mathbf{1}=y^{-1} y$. Furthermore, let $q$ be a primitive root of unity of degree three, so that $q^{3}=1$ and $1+q+q^{2}=0$. Then

$$
\left(\phi_{1} f\right)(x, y)=f\left(q x, q^{2} y\right), \quad\left(\phi_{2} f\right)(x, y)=\left(\phi_{1}^{2} f\right)(x, y)=f\left(q^{2} x, q y\right)
$$

determines a faithful action of $\mathbb{Z}_{3}$ on $\mathcal{A}$ by automorphisms. In the associated differential calculus we have

$$
\theta^{1} f(x, y)=f\left(q x, q^{2} y\right) \theta^{1}, \quad \theta^{2} f(x, y)=f\left(q^{2} x, q y\right) \theta^{2}
$$


and, using (4.12) with (4.1) and $t_{1}=t_{2}=q-1$,

$$
\mathrm{d} x=x \theta^{1}-q^{2} x \theta^{2}, \quad \mathrm{~d} y=-q^{2} y \theta^{1}+y \theta^{2} .
$$

This implies

$$
\theta^{1}=\frac{1}{1-q}\left(x^{-1} \mathrm{~d} x+q^{2} y^{-1} \mathrm{~d} y\right), \quad \theta^{2}=\frac{1}{1-q}\left(q^{2} x^{-1} \mathrm{~d} x+y^{-1} \mathrm{~d} y\right)
$$

and

$$
\begin{aligned}
\mathrm{d} x x & =-x \mathrm{~d} x+x^{2} y^{-1} \mathrm{~d} y, & \mathrm{~d} x y & =-x \mathrm{~d} y, \\
\mathrm{~d} y y & =-y \mathrm{~d} y+y^{2} x^{-1} \mathrm{~d} x, & \mathrm{~d} y x & =-y \mathrm{~d} x .
\end{aligned}
$$

Using these relations and the Leibniz rule, we find

$$
\mathrm{d}\left(x^{2}\right)=x^{2} y^{-1} \mathrm{~d} y, \quad \mathrm{~d}\left(y^{2}\right)=y^{2} x^{-1} \mathrm{~d} x,
$$

and

$$
\mathrm{d}\left(x^{3}\right)=\mathrm{d}\left(y^{3}\right)=\mathrm{d}(x y)=\mathrm{d}(y x)=0 .
$$

As a consequence,

$$
x^{3}=c_{1}, \quad y^{3}=c_{2}, \quad x y=c_{3}, \quad y x=c_{4},
$$

are constants of the calculus, i.e., $\phi_{s}\left(c_{i}\right)=c_{i}, s=1,2$ and $i=1,2,3,4$. Furthermore, we find

$$
\mathrm{d}\left(x^{2}\right)=x^{3}(y x)^{-1} \mathrm{~d} y=c_{1} c_{4}^{-1} \mathrm{~d} y, \quad \mathrm{~d}\left(y^{2}\right)=y^{3}(x y)^{-1} \mathrm{~d} x=c_{2} c_{3}^{-1} \mathrm{~d} x,
$$

and thus

$$
\mathrm{d}\left(x^{2}-c_{1} c_{4}^{-1} y\right)=0, \quad \mathrm{~d}\left(y^{2}-c_{2} c_{3}^{-1} x\right)=0,
$$

so we can set $x^{2}-c_{1} c_{4}^{-1} y=c_{5}$ and $y^{2}-c_{2} c_{3}^{-1} x=c_{6}$. Multiplying the first equation with $x$ and the second with $y$ from the right leads to $c_{5}=0=c_{6}$. Hence

$$
x^{2}=c_{1} c_{4}^{-1} y, \quad y^{2}=c_{2} c_{3}^{-1} x .
$$

It follows that every $f \in \mathcal{A}$ can be written as a linear combination of $\mathbf{1}, x, y$, where the coefficients are constants with respect to the differential calculus.

If we divide $\mathcal{A}$ by the ideal generated by $c_{1}-\mathbf{1}, c_{2}-\mathbf{1}, c_{3}-\mathbf{1}, c_{4}-\mathbf{1}$, we obtain an algebra $\mathcal{A}^{\prime}$ generated by $x$ with the relation $x^{3}=\mathbf{1}$. The elements

$$
e^{0}=\frac{1}{3}\left(\mathbf{1}+x+x^{2}\right), \quad e^{1}=\frac{1}{3}\left(\mathbf{1}+q^{2} x+q x^{2}\right), \quad e^{2}=\frac{1}{3}\left(\mathbf{1}+q x+q^{2} x^{2}\right)
$$

of $\mathcal{A}^{\prime}$ are then primitive idempotents and satisfy $e^{0}+e^{1}+e^{2}=1$. As a consequence, $\mathcal{A}^{\prime}$ becomes the algebra of functions on $\mathbb{Z}_{3}$ and the above differential calculus is the universal one. 
The maps $\phi_{s}$ obviously preserve the relations (4.62) in the sense of (3.4) and are therefore differentiable. (4.61) then implies $\phi_{s}\left(\theta^{s^{\prime}}\right)=\theta^{s^{\prime}}$.

Example 4.8. Let $\mathcal{A}$ be a noncommutative matrix algebra and $G$ a group which acts faithfully on $\mathcal{A}$ by inner automorphisms $\left\{\phi_{g} \mid g \in G\right\}$, so that $\phi_{g}(f)=U_{g} f U_{g}^{-1}$ with an invertible matrix $U_{g}$. Each set $S \subset G \backslash\{e\}$ then determines a differential calculus. If we have 1-parameter groups $U_{s}=e^{t_{s} \lambda_{s}}$ with matrices $\lambda_{s}$ and $t_{s} \in \mathbb{R}$, in the limit $t_{s} \rightarrow 0$ we obtain $\theta^{s} f=f \theta^{s}$ and

$$
\lim _{t_{s} \rightarrow 0} e_{s} f=\lim _{t_{s} \rightarrow 0} \frac{\phi_{s}(f)-f}{t_{s}}=\left[\lambda_{s}, f\right]
$$

so that each $e_{s}$ tends to a derivation, a special case of (5.1). Assuming $U_{s}^{-1} \in \mathcal{A}$, (4.10) implies that $U_{s}^{-1} \theta^{s}, s \in S$, are 1-forms which commute with all $f \in \mathcal{A}$ (see also Lemma 4.11). Then we can modify $\vartheta$ as follows,

$$
\vartheta^{\prime}=\vartheta-\sum_{s \in S} \frac{1}{t_{s}} U_{s}^{-1} \theta^{s}=\sum_{s \in S} \frac{\mathbf{1}-U_{s}^{-1}}{t_{s}} \theta^{s}
$$

while keeping $\mathrm{d} f=\left[\vartheta^{\prime}, f\right]$. In contrast to $\vartheta$, the 1 -form $\vartheta^{\prime}$ has a limit:

$$
\lim _{\left\{t_{s}\right\} \rightarrow 0} \vartheta^{\prime}=\sum_{s \in S} \lambda_{s} \theta^{s} .
$$

The differential calculi obtained in this limit have been explored in Ref. 7. They are not reached directly by the construction presented in the main part of this section and demand a suitable generalization which is the subject of the following section.

\section{Differential calculi associated with twisted inner deriva- tions}

Example 4.8 (see also Ref. 17) demonstrates that the construction of differential calculi associated with automorphisms presented in section 4 does not exhaust the possibilities offered by (1.2) and suggests a suitable generalization. Let $\phi_{s}$ be automorphisms of $\mathcal{A}$ and $\lambda_{s} \in \mathcal{A}$. We introduce "twisted inner derivations" by

$$
e_{s}(f)=\lambda_{s} \phi_{s}(f)-f \lambda_{s} \quad \forall f \in \mathcal{A}
$$

with $\lambda_{s} \in \mathcal{A}$. They satisfy

$$
e_{s}\left(f f^{\prime}\right)=e_{s}(f) \phi_{s}\left(f^{\prime}\right)+f e_{s}\left(f^{\prime}\right) \quad \forall f, f^{\prime} \in \mathcal{A} .
$$

Using the relations (4.3) and (4.4), we obtain an $\mathcal{A}$-bimodule $\mathcal{X}$ and via (4.5)-(4.7) a dual module $\hat{\Omega}^{1}(\mathcal{A})$. If $\left\{e_{s} \mid s \in S\right\}$ is a basis of $\mathcal{X}$, let $\left\{\theta^{s} \mid s \in S\right\}$ be the dual basis. Then (1.2) holds and

$$
\mathrm{d} f=[\vartheta, f]=\sum_{s \in S} e_{s}(f) \theta^{s}
$$


with

$$
\vartheta=\sum_{s} \lambda_{s} \theta^{s}
$$

defines a first order differential calculus $\left(\hat{\Omega}^{1}(\mathcal{A}), \mathrm{d}\right)$. All formulas and results of section 4 , with the exception of the subsections, remain valid after replacing (4.11) by (5.1). We only have to exchange (4.15) with (5.4) and note that now $c$ is a constant of the differential calculus iff $c \lambda_{s}=\lambda_{s} \phi_{s}(c)$ for all $s \in S$.

Example 5.1. The defining relations of the quantum plane differential calculus of example 4.4 can be presented in various ways if we relax (4.1) and allow twisted derivations, while keeping the structure (1.2). Let us look more closely at the covariant calculus (4.41) where $\theta^{\hat{1}}(x, y)=r^{-1}(x, y) \theta^{\hat{1}}$ and $\theta^{\hat{2}}(x, y)=\left(x, r^{-1} y\right) \theta^{\hat{2}}$ with $r=p q$. Let $\theta^{1}:=x^{k} y^{l} \theta^{\hat{1}}$ and $\theta^{2}:=x^{m} y^{n} \theta^{\hat{2}}$, so that $\lambda_{1}=y^{-l} x^{-k} / t_{\hat{1}}, \lambda_{2}=y^{-n} x^{-m} / t_{\hat{2}}$, assuming that $x, y$ are invertible. Then $\theta^{1}(x, y)=r^{-1}\left(q^{-l} x, q^{k} y\right) \theta^{1}(k, l), \theta^{2}(x, y)=\left(q^{-n} x, r q^{m} y\right) \theta^{2}$. In particular, if $p=q$ we can choose $k=2, l=-2, m=2, n=0$, and the 1 -forms $\theta^{1}$ and $\theta^{2}$ commute with all elements of $\mathcal{A}$ (see also Ref. 7). In the latter case, (5.1) become ordinary inner derivations and the automorphism structure is hidden.

In the first subsection we explore the structure of 2-forms. The second subsection reveals the above structure in a bicovariant differential calculus on the quantum group $G L_{p, q}(2)$.

\subsection{The structure of 2 -forms}

Using (2.5) and (5.4), we find

$$
\zeta=\sum_{s \in S} \lambda_{s} \mathrm{~d} \theta^{s}-\sum_{s, s^{\prime} \in S} \lambda_{s^{\prime}} \lambda_{s} \theta^{s} \theta^{s^{\prime}}=-\sum_{s \in S} \lambda_{s} \Delta\left(\theta^{s}\right)+\sum_{s, s^{\prime} \in S} \lambda_{s} \phi_{s}\left(\lambda_{s^{\prime}}\right) \theta^{s} \theta^{s^{\prime}} .
$$

Commuting $f \in \mathcal{A}$ from the right towards the left of each term in this formula, using (1.2) and (2.6), leads to

$$
\sum_{s, s^{\prime} \in S}\left(f \lambda_{s} \phi_{s}\left(\lambda_{s^{\prime}}\right)-\lambda_{s} \phi_{s}\left(\lambda_{s^{\prime}}\right) \phi_{s s^{\prime}}(f)\right) \theta^{s} \theta^{s^{\prime}}=\sum_{s \in S}\left(f \lambda_{s}-\lambda_{s} \phi_{s}(f)\right) \Delta\left(\theta^{s}\right) .
$$

Its elaboration in concrete examples yields the 2-form relations and determines the map $\Delta$.

Let $\Xi$ be a subset of $S \times S$ such that $\left\{\theta^{s} \theta^{s^{\prime}} \mid\left(s, s^{\prime}\right) \in \Xi\right\}$ is a left $\mathcal{A}$-module basis of $\hat{\Omega}^{2}(\mathcal{A})$. Writing

$$
\zeta=\sum_{\left(s, s^{\prime}\right) \in \Xi} \zeta_{s, s^{\prime}} \theta^{s} \theta^{s^{\prime}}
$$

(2.6) demands

$$
f \zeta_{s, s^{\prime}}=\phi_{s s^{\prime}}(f) \zeta_{s, s^{\prime}} \quad \forall f \in \mathcal{A},\left(s, s^{\prime}\right) \in \Xi
$$

Example 5.2. Let us consider the Heisenberg algebra generated by $x, y$ with $[x, y]=1$. We choose $\phi_{s}=\mathrm{id}, s=1,2$, and $\lambda_{1}=-y, \lambda_{2}=x$. Then $e_{s} f=\left[\lambda_{s}, f\right], \theta^{1}=\mathrm{d} x, \theta^{2}=\mathrm{d} y$, 
$\theta^{s} f=f \theta^{s}$, and $\vartheta=x \mathrm{~d} y-y \mathrm{~d} x$. Evaluation of (5.6) leads to the 2-form relations $\left(\theta^{s}\right)^{2}=0$, $\theta^{2} \theta^{1}=-\theta^{1} \theta^{2}$, and moreover requires $\Delta\left(\theta^{s}\right)=0$. Hence we may choose $\Xi=\{(1,2)\}$. Furthermore, (2.5) shows that $\zeta=\theta^{1} \theta^{2}$.

If we add one more derivation with $\lambda_{3}=y x$, there is a third 1-form $\theta^{3}$. Inspection of (5.6) then yields $\Delta\left(\theta^{1}\right)=-\theta^{1} \theta^{3}, \Delta\left(\theta^{2}\right)=\theta^{2} \theta^{3}, \Delta\left(\theta^{3}\right)=-\theta^{1} \theta^{2}-\theta^{2} \theta^{1}$, and the 2-form relations $\left(\theta^{1}\right)^{2}=\left(\theta^{2}\right)^{2}=\left(\theta^{3}\right)^{2}=0, \theta^{3} \theta^{1}=-\theta^{1} \theta^{3}, \theta^{3} \theta^{2}=-\theta^{2} \theta^{3}$. Hence we can choose $\Xi=\{(1,2),(2,1),(1,3),(2,3)\}$. Furthermore, $\zeta=-\theta^{2} \theta^{1}$.

\subsection{A bicovariant calculus on $G L_{p, q}(2)$}

The quantum group $G L_{p, q}(2)$ is given by the algebra $\mathcal{A}$ generated by elements $a, b, c, d$ with relations

$$
\begin{aligned}
& a b=p b a, \quad a c=q c a, \quad b c=(q / p) c b, \quad b d=q d b, \quad c d=p d c \\
& a d=d a+\left(p-q^{-1}\right) b c
\end{aligned}
$$

and carries a Hopf algebra structure. ${ }^{7}$ Its automorphisms are given by scalings $(a, b, c, d) \mapsto$ $(\alpha a, \beta b, \gamma c, \delta d)$ with $\alpha, \beta, \gamma, \delta \in \mathbb{C} \backslash\{0\}$ such that $\alpha \delta=\beta \gamma .^{8}$ Of course, we can use these automorphisms to construct differential calculi on $G L_{p, q}(2)$ according to the prescription of section 4. An interesting question is whether the distinguished bicovariant differential calculi on $G L_{p, q}(2)$ [10] are of this form or at least exhibit the more general structure described in the beginning of this section. Let us look more closely at one of them which is determined in terms of generalized Maurer-Cartan 1 -forms $\tilde{\theta}^{i}, i=1, \ldots, 4$, by the relations

$$
\begin{aligned}
& \tilde{\theta}^{1} a=r a \tilde{\theta}^{1}+(r-1) b \tilde{\theta}^{3}, \quad \tilde{\theta}^{2} a=q a \tilde{\theta}^{2}+p^{-1}(r-1) b \tilde{\theta}^{4}, \quad \tilde{\theta}^{3} a=p a \tilde{\theta}^{3}, \quad \tilde{\theta}^{4} a=a \tilde{\theta}^{4}, \\
& \tilde{\theta}^{1} b=b \tilde{\theta}^{1}+(r-1) a \tilde{\theta}^{2}+r^{-1}(r-1)^{2} b \tilde{\theta}^{4}, \quad \tilde{\theta}^{2} b=q b \tilde{\theta}^{2}, \\
& \tilde{\theta}^{3} b=p b \tilde{\theta}^{3}+q^{-1}(r-1) a \tilde{\theta}^{4}, \quad \tilde{\theta}^{4} b=r b \tilde{\theta}^{4}, \\
& \tilde{\theta}^{1} c=r c \tilde{\theta}^{1}+(r-1) d \tilde{\theta}^{3}, \quad \tilde{\theta}^{2} c=q c \tilde{\theta}^{2}+p^{-1}(r-1) d \tilde{\theta}^{4}, \quad \tilde{\theta}^{3} c=p c \tilde{\theta}^{3}, \quad \tilde{\theta}^{4} c=c \tilde{\theta}^{4}, \\
& \tilde{\theta}^{1} d=d \tilde{\theta}^{1}+(r-1) c \tilde{\theta}^{2}+r^{-1}(r-1)^{2} d \tilde{\theta}^{4}, \quad \tilde{\theta}^{2} d=q d \tilde{\theta}^{2}, \\
& \tilde{\theta}^{3} d=p d \tilde{\theta}^{3}+q^{-1}(r-1) c \tilde{\theta}^{4}, \quad \tilde{\theta}^{4} d=r d \tilde{\theta}^{4}
\end{aligned}
$$

where $r=p q$ (see [10], example 1 of section 6). The action of the exterior derivative $\mathrm{d}$ on $G L_{p, q}(2)$ is determined by

$$
\mathrm{d} a=a \tilde{\theta}^{1}+b \tilde{\theta}^{3}, \quad \mathrm{~d} b=a \tilde{\theta}^{3}+b \tilde{\theta}^{4}, \quad \mathrm{~d} c=c \tilde{\theta}^{1}+d \tilde{\theta}^{3}, \quad \mathrm{~d} d=c \tilde{\theta}^{2}+d \tilde{\theta}^{4} .
$$

Let us introduce

$$
\theta^{4}=k_{4} \mathcal{D}^{P} b^{N} c^{M} \tilde{\theta}^{4}
$$

\footnotetext{
${ }^{7}$ The existence of an antipode requires that the quantum determinant $\mathcal{D}$ (see below) is invertible. In most of what follows we will not need this condition, however.

${ }^{8}$ If $p=q$, in which case the quantum group is denoted as $G L_{q}(2)$, there is an additional automorphism which interchanges $b$ and $c$, and leaves $a, d$ fixed [15].
} 
with $k_{4} \in \mathbb{C} \backslash\{0\}, \mathcal{D}=a d-p b c$ and arbitrary non-negative integers $M, N, P .{ }^{9}$ Then (1.2) holds for $\theta^{4}$ with the automorphism $\phi_{4}$ which acts as follows on the generators:

$$
\begin{array}{ll}
\phi_{4}(a)=p^{-N} q^{-M} a, & \phi_{4}(b)=r(p / q)^{M+P} b, \\
\phi_{4}(c)=(q / p)^{N+P} c, \quad \phi_{4}(d)=p^{M+1} q^{N+1} d .
\end{array}
$$

Moreover,

$$
\theta^{2}=k_{2} \mathcal{D}^{Q} b^{K} c^{L}\left(c \tilde{\theta}^{2}+d \tilde{\theta}^{4}\right)
$$

with $k_{2} \in \mathbb{C} \backslash\{0\}$ and non-negative integers $Q, K, L$ also satisfies (1.2) with

$$
\begin{aligned}
& \phi_{2}(a)=p^{-K} q^{-L} a, \quad \phi_{2}(b)=p^{Q+L+1} q^{-Q-L} b \\
& \phi_{2}(c)=p^{-Q-K} q^{Q+K+1} c, \quad \phi_{2}(d)=p^{L+1} q^{K+1} d .
\end{aligned}
$$

Furthermore,

$$
\theta^{3}=k_{3} \mathcal{D}^{R} b^{S} c^{T}\left(b \tilde{\theta}^{3}-r^{-1} a \tilde{\theta}^{4}\right)
$$

with $k_{3} \in \mathbb{C} \backslash\{0\}$ and non-negative integers $R, S, T$ satisfies (1.2) with

$$
\begin{aligned}
& \phi_{3}(a)=p^{-S} q^{-T} a, \quad \phi_{3}(b)=p^{R+T+1} q^{-R-T} b, \\
& \phi_{3}(c)=p^{-R-S} q^{R+S+1} c, \quad \phi_{3}(d)=p^{T+1} q^{S+1} d .
\end{aligned}
$$

Finally,

$$
\theta^{1}=k_{1} \mathcal{D}^{U} b^{V} c^{W}\left(b c \tilde{\theta}^{1}-p^{-1} a c \tilde{\theta}^{2}+b d \tilde{\theta}^{3}-p^{-1} a d \tilde{\theta}^{4}\right)
$$

with $k_{1} \in \mathbb{C} \backslash\{0\}$ and non-negative integers $U, V, W$ satisfies (1.2) with

$$
\begin{aligned}
& \phi_{1}(a)=p^{-V} q^{-W} a, \quad \phi_{1}(b)=(p / q)^{U+W+1} b \\
& \phi_{1}(c)=q^{2}(q / p)^{U+V} c, \quad \phi_{1}(d)=p^{W+1} q^{V+1} d .
\end{aligned}
$$

Hence, in terms of the new 1-forms $\theta^{s}$ the commutation relations of the above bicovariant differential calculus on $G L_{p, q}(2)$ indeed take the form (1.2) and thus have the "automorphism structure" we were looking for. This is quite surprising and raises the question to what extent this result generalizes to other bicovariant differential calculi on quantum groups (see [11, in particular).

In order to express the Maurer-Cartan 1-forms $\tilde{\theta}^{s}$ as ordinary 1-forms, we need $\mathcal{D}$ to be invertible:

$$
\left(\begin{array}{cc}
\tilde{\theta}^{1} & \tilde{\theta}^{2} \\
\tilde{\theta}^{3} & \tilde{\theta}^{4}
\end{array}\right)=\mathcal{D}^{-1}\left(\begin{array}{cc}
d \mathrm{~d} a-q^{-1} b \mathrm{~d} c & d \mathrm{~d} b-q^{-1} b \mathrm{~d} d \\
-q c \mathrm{~d} a+a \mathrm{~d} c & -q c \mathrm{~d} b+a \mathrm{~d} d
\end{array}\right)
$$

Let us write $\left(a_{s}\right)=(a, b, c, d)$ and $\phi_{s}\left(a_{s^{\prime}}\right)=\alpha_{s, s^{\prime}} a_{s^{\prime}}$ where $\alpha_{s, s^{\prime}} \in \mathbb{C}$. Using (15.20) we find that

$$
\phi_{s}\left(\tilde{\theta}^{1}\right)=\tilde{\theta}^{1}, \quad \phi_{s}\left(\tilde{\theta}^{2}\right)=\left(\alpha_{s, 2} / \alpha_{s, 1}\right) \tilde{\theta}^{2}, \quad \phi_{s}\left(\tilde{\theta}^{3}\right)=\left(\alpha_{s, 3} / \alpha_{s, 4}\right) \tilde{\theta}^{3}, \quad \phi_{s}\left(\tilde{\theta}^{4}\right)=\tilde{\theta}^{4}
$$

\footnotetext{
${ }^{9}$ Note that $\mathcal{D} a=a \mathcal{D}, \mathcal{D} b=(p / q) b \mathcal{D}, \mathcal{D} c=(q / p) c \mathcal{D}, \mathcal{D} d=d \mathcal{D}$. The assumption of non-negative exponents can be relaxed if the corresponding elements of $\mathcal{A}$ are assumed to be invertible.
} 
must hold for $\phi_{s}$ to be differentiable. ${ }^{10}$ The maps $\phi_{s}$ then preserve the relations (15.10) and are indeed differentiable. The action of $\phi_{s}$ on the $\theta^{s}$ is now easily obtained and also turns out to be homogeneous, i.e., it is given by scalings with complex numbers. The calculus is inner at first order and simple, but the 1-form

$$
\vartheta=(r-1)^{-1}\left(\tilde{\theta}^{1}+r^{-1} \tilde{\theta}^{4}\right)
$$

is not given by the formula (4.15).

To be more explicit, let us choose

$$
\begin{aligned}
& \theta^{1}=(r-1)^{-1} c^{-1} b^{-1}\left(b c \tilde{\theta}^{1}-p^{-1} a c \tilde{\theta}^{2}+b d \tilde{\theta}^{3}-p^{-1} a d \tilde{\theta}^{4}\right) \\
& \theta^{2}=q(r-1)^{-1} c^{-1} b^{-1}\left(c \tilde{\theta}^{2}+d \tilde{\theta}^{4}\right) \\
& \theta^{3}=-[p(r-1)]^{-1} c^{-1} b^{-1}\left(b \tilde{\theta}^{3}-r^{-1} a \tilde{\theta}^{4}\right) \\
& \theta^{4}=-[p(r-1)]^{-1} c^{-1} b^{-1} \mathcal{D} \tilde{\theta}^{4}
\end{aligned}
$$

assuming that $b$ and $c$ are invertible. Then we obtain

$$
\vartheta=\theta^{1}+a \theta^{2}+d \theta^{3}+\theta^{4}
$$

and thus

$e_{1}(f)=\phi_{1}(f)-f, e_{2}(f)=a \phi_{2}(f)-f a, e_{3}(f)=d \phi_{3}(f)-f d, e_{4}(f)=\phi_{4}(f)-f,(5$.

where the action of the automorphisms $\phi_{s}$ on the quantum group algebra is determined by

$$
\left(\alpha_{s, s^{\prime}}\right)=\left(\begin{array}{llll}
r & 1 & r & 1 \\
r & q & p & 1 \\
r & q & p & 1 \\
r & r & 1 & 1
\end{array}\right)
$$

Furthermore,

$$
\phi_{s}\left(\theta^{1}\right)=\theta^{1}, \quad \phi_{s}\left(\theta^{2}\right)=r^{-1} \theta^{2}, \quad \phi_{s}\left(\theta^{3}\right)=\theta^{3}, \quad \phi_{s}\left(\theta^{4}\right)=\theta^{4},
$$

which implies $\phi_{s}(\vartheta)=\vartheta$, in accordance with Lemma 3.1.

The bicovariant differential calculus extends to higher orders in such a way that

$$
\mathrm{d} \omega=[\vartheta, \omega]
$$

for an arbitrary form $\omega \cdot{ }^{11}$ Hence $\Delta(\omega)=0$ and $\zeta=\vartheta^{2}$.

\footnotetext{
${ }^{10}$ Note that $\alpha_{s, 1} \alpha_{s, 4}=\alpha_{s, 2} \alpha_{s, 3}$.

${ }^{11}$ The generalized wedge product has been obtained in Ref. 10, see (6.27) therein.
} 


\section{Connections}

A connection on a left $\mathcal{A}$-module $\mathfrak{E}$ is a linear map $\nabla: \mathfrak{E} \rightarrow \hat{\Omega}^{1}(\mathcal{A}) \otimes_{\mathcal{A}} \mathfrak{E}$ such that

$$
\nabla(f E)=\mathrm{d} f \otimes_{\mathcal{A}} E+f \nabla(E) \quad \forall E \in \mathfrak{E}
$$

with respect to a (possibly generalized) differential calculus $(\hat{\Omega}(\mathcal{A})$, d). It extends to a map $\nabla: \hat{\Omega}(\mathcal{A}) \otimes_{\mathcal{A}} \mathfrak{E} \rightarrow \hat{\Omega}(\mathcal{A}) \otimes_{\mathcal{A}} \mathfrak{E}$ via

$$
\nabla\left(\omega \otimes_{\mathcal{A}} E\right)=\mathrm{d} \omega \otimes_{\mathcal{A}} E+(-1)^{r} \omega \nabla E \quad \forall \omega \in \hat{\Omega}^{r}(\mathcal{A}), E \in \mathfrak{E} .
$$

Its curvature is the left $\mathcal{A}$-module homomorphism $\mathcal{R}: \mathfrak{E} \rightarrow \hat{\Omega}^{2}(\mathcal{A}) \otimes_{\mathcal{A}} \mathfrak{E}$ given by

$$
\mathcal{R}(E)=-\nabla^{2} E
$$

which extends to a map $\hat{\Omega}(\mathcal{A}) \otimes_{\mathcal{A}} \mathfrak{E} \rightarrow \hat{\Omega}(\mathcal{A}) \otimes_{\mathcal{A}} \mathfrak{E}$ with the property

$$
\mathcal{R}\left(\omega \otimes_{\mathcal{A}} E\right)=\omega \mathcal{R}(E) \quad \forall \omega \in \hat{\Omega}(\mathcal{A}), E \in \mathfrak{E} .
$$

Lemma 6.1 Let $\mathrm{d}: \mathcal{A} \rightarrow \hat{\Omega}^{1}(\mathcal{A})$ be inner. Every connection on $\mathfrak{E}$ is then of the form

$$
\nabla(E)=\vartheta \otimes_{\mathcal{A}} E-\mathcal{V}(E) \quad \forall E \in \mathfrak{E}
$$

where $\mathcal{V}: \mathfrak{E} \rightarrow \hat{\Omega}^{1} \otimes_{\mathcal{A}} \mathfrak{E}$ satisfies

$$
\mathcal{V}(f E)=f \mathcal{V}(E) \quad \forall f \in \mathcal{A} .
$$

Conversely, every linear map $\mathcal{V}$ with this property defines a connection via the above formula.

Proof: This is easily verified using (2.1) (see also Ref. [5).

Now let $(\hat{\Omega}(\mathcal{A})$, d) be a (generalized) differential calculus which is inner at first order and associated with automorphisms $\left\{\phi_{s} \mid s \in S\right\}$ of $\mathcal{A}$ such that (1.2) holds. Writing

$$
\mathcal{V}=\sum_{s \in S} \theta^{s} \otimes_{\mathcal{A}} \mathcal{V}_{s}
$$

with parallel transport operators $\mathcal{V}_{s}$ ("in the $s$-direction"), we find

$$
\mathcal{V}_{s}(f E)=\phi_{s}^{-1}(f) \mathcal{V}_{s}(E) .
$$

\subsection{Linear connections}

If $\mathfrak{E}=\hat{\Omega}^{1}(\mathcal{A}), \nabla$ is called a linear connection. The torsion of $\nabla$ is the left $\mathcal{A}$-module homomorphism $\Theta: \hat{\Omega}^{1}(\mathcal{A}) \rightarrow \hat{\Omega}^{2}(\mathcal{A})$ defined by

$$
\Theta(\alpha)=\mathrm{d} \alpha-\pi \circ \nabla \alpha \quad \forall \alpha \in \hat{\Omega}^{1}(\mathcal{A})
$$

where $\pi$ is the canonical projection $\hat{\Omega}^{1}(\mathcal{A}) \otimes_{\mathcal{A}} \hat{\Omega}^{1}(\mathcal{A}) \rightarrow \hat{\Omega}^{2}(\mathcal{A})$. It extends to a map $\Theta$ : $\hat{\Omega}(\mathcal{A}) \otimes_{\mathcal{A}} \hat{\Omega}^{1}(\mathcal{A}) \rightarrow \hat{\Omega}(\mathcal{A})$ via

$$
\Theta=\mathrm{d} \circ \pi-\pi \circ \nabla
$$


with the property

$$
\Theta\left(\omega \otimes_{\mathcal{A}} \alpha\right)=(-1)^{r} \omega \Theta(\alpha)
$$

for all $\alpha \in \hat{\Omega}^{1}(\mathcal{A})$ and $\omega \in \hat{\Omega}^{r}(\mathcal{A})$. Here $\pi$ denotes more generally the canonical projection $\hat{\Omega}(\mathcal{A}) \otimes_{\mathcal{A}} \hat{\Omega}^{1}(\mathcal{A}) \rightarrow \hat{\Omega}(\mathcal{A})$.

If $(\hat{\Omega}(\mathcal{A}), \mathrm{d})$ is a (generalized) differential calculus associated with automorphisms $\left\{\phi_{s} \mid s \in\right.$ $S\}$ of $\mathcal{A}$, we set

$$
\mathcal{V}_{s}\left(\theta^{s^{\prime}}\right)=\sum_{s^{\prime \prime} \in S} \phi_{s}^{-1}\left(V_{s, s^{\prime \prime}}^{s^{\prime}}\right) \theta^{s^{\prime \prime}}
$$

with $V_{s, s^{\prime \prime}}^{s^{\prime}} \in \mathcal{A}$. If the differential calculus is of the kind described in section 4, using (4.15), (4.26), (6.5) and (6.7), the torsion is given by

$$
\Theta\left(\theta^{s}\right)=\theta^{s} \vartheta-\Delta\left(\theta^{s}\right)+\sum_{s^{\prime} \in S} \theta^{s^{\prime}} \mathcal{V}_{s^{\prime}}\left(\theta^{s}\right)=\sum_{s^{\prime}, s^{\prime \prime} \in S}\left(\delta_{s^{\prime}}^{s}-\delta_{s^{\prime} s^{\prime \prime}}^{s}+V_{s^{\prime}, s^{\prime \prime}}^{s}\right) \theta^{s^{\prime}} \theta^{s^{\prime \prime}} .
$$

Here we have chosen $t_{s}=1$ for simplicity. According to the decomposition of a 2-form into biangle, triangle and quadrangle parts, the condition of vanishing torsion splits into three sets of equations. The biangle torsion vanishes iff

$$
V_{s^{\prime}, s^{\prime \prime}}^{s}=-\delta_{s^{\prime}}^{s} \mathbf{1} \quad \forall s^{\prime}, s^{\prime \prime} \in S \text { with } s^{\prime} s^{\prime \prime}=e .
$$

The vanishing of the triangle part of the torsion 2-form amounts to

$$
V_{s^{\prime}, s^{\prime \prime}}^{s}=\left(\delta_{s^{\prime} s^{\prime \prime}}^{s}-\delta_{s^{\prime}}^{s}\right) \mathbf{1} \quad \forall s^{\prime}, s^{\prime \prime} \in S \text { with } s^{\prime} s^{\prime \prime} \in S .
$$

In particular, this restricts the biangle and triangle components of $V_{s^{\prime}, s^{\prime \prime}}^{s}$ to values in $\mathbb{k}$. In reading off the quadrangle torsion components, one has to take care of the 2-form relations (4.25). Since all these relations are linear in $V_{s^{\prime}, s^{\prime \prime}}^{s}$, they do not feel the algebra structure. In case of vanishing torsion, only some of the quadrangle components of $V_{s^{\prime}, s^{\prime \prime}}^{s}$ could have values in $\mathcal{A} \backslash \mathbb{k} \mathbf{1}$.

We should also mention that the condition of vanishing torsion has not the distinguished status as in continuum differential (Riemannian) geometry, as demonstrated in Ref. 6.

Example 6.1. In example 4.4 we constructed differential calculi from $\mathbb{Z}^{2}$ automorphism actions on the quantum plane algebra. With respect to such a calculus, a linear connection on the quantum plane is torsion-free iff $V_{\hat{2}, \hat{1}}^{\hat{1}}=V_{\hat{1}, \hat{2}}^{\hat{1}}+1$ and $V_{\hat{2}, \hat{1}}^{\hat{2}}=V_{\hat{1}, \hat{2}}^{\hat{2}}-1$. These are precisely the relations which we obtained for the group lattice $\left(\mathbb{Z}^{2}, S=\{\hat{1}, \hat{2}\}\right)$ in Ref. 6. section 4.2.2. The only difference is that now $V_{s^{\prime}, s^{\prime \prime}}^{s}$ takes values more generally in the quantum plane algebra.

\section{Noncommutative geometries associated with differ- entiable automorphisms}

In this section we assume that $\left\{\phi_{s}, \phi_{s}^{-1} \mid s \in S\right\}$ is a set of differentiable automorphisms of $\mathcal{A}$. Let $(\hat{\Omega}(\mathcal{A}), \mathrm{d})$ be a differential calculus which is inner at first order and satisfies (1.2) with respect to a basis $\theta^{s}$ of $\hat{\Omega}^{1}(\mathcal{A})$. 


\subsection{A semi-left-linear tensor product}

Let $T$ an arbitrary element of an $n$-fold tensor product space $\left(\hat{\Omega}^{1}\right)^{\otimes n}:=\hat{\Omega}^{1}(\mathcal{A}) \otimes_{\mathcal{A}} \ldots \otimes_{\mathcal{A}}$ $\hat{\Omega}^{1}(\mathcal{A})$. Then a new tensor product can be defined via

$$
\theta^{s} \otimes_{L} T:=\theta^{s} \otimes_{\mathcal{A}} \phi_{s}^{-1} T .
$$

This product is associative and has the following semi-left-linearity property (with respect to the 1 -form basis $\theta^{s}$ ):

$$
f \theta^{s} \otimes_{L}\left(f^{\prime} T\right)=f f^{\prime} \theta^{s} \otimes_{L} T .
$$

If $\mathcal{A}$ is not commutative, this product is not left $\mathcal{A}$-linear since $\alpha \otimes_{L}(f T) \neq f \alpha \otimes_{L} T$ for $\alpha \in \hat{\Omega}^{1}$ and $f \in \mathcal{A}$, in general.

\subsection{Metric and compatibility with a linear connection}

Let $\nabla$ be a linear connection with transport operators $\mathcal{V}_{s}$. The following procedure extends it to a connection on $\hat{\Omega}^{1}(\mathcal{A}) \otimes_{L} \hat{\Omega}^{1}(\mathcal{A})$ (and more generally on $n$-fold semi-left-linear tensor products). We define

$$
\mathcal{V}_{s}\left(\alpha \otimes_{L} \beta\right):=\mathcal{V}_{s}(\alpha) \otimes_{L} \mathcal{V}_{s}(\beta)
$$

for all $\alpha, \beta \in \hat{\Omega}^{1}(\mathcal{A})$. The new $\mathcal{V}_{s}$ satisfy (6.8) and define via (6.7) a left $\mathcal{A}$-module homomorphism $\hat{\Omega}^{1}(\mathcal{A}) \otimes_{L} \hat{\Omega}^{1}(\mathcal{A}) \rightarrow \hat{\Omega}^{1}(\mathcal{A}) \otimes_{\mathcal{A}}\left(\hat{\Omega}^{1}(\mathcal{A}) \otimes_{L} \hat{\Omega}^{1}(\mathcal{A})\right)$ and thus, according to Lemma 6.1] a connection which we also denote as $\nabla .{ }^{12}$

A metric is taken to be an element $g \in \hat{\Omega}^{1}(\mathcal{A}) \otimes_{L} \hat{\Omega}^{1}(\mathcal{A})$, i.e.,

$$
\mathrm{g}=\sum_{s, s^{\prime} \in S} \mathfrak{g}_{s, s^{\prime}} \theta^{s} \otimes_{L} \theta^{s^{\prime}}
$$

with a matrix of coefficients $\mathfrak{g}_{s, s^{\prime}} \in \mathcal{A}$ subject to suitable restrictions. There is a distinguished class of metrics which are invariant under the action of all the automorphisms $\phi_{s}$, i.e.

$$
\phi_{s}(\mathrm{~g})=\mathrm{g} \quad \forall s \in S
$$

A linear connection $\nabla$ is compatible with the metric $g$ if

$$
\nabla \mathrm{g}=0
$$

This is equivalent to $\mathcal{V}_{s}(\mathrm{~g})=\mathrm{g}$ for all $s \in S$. Using (6.12), it becomes

$$
\phi_{s}\left(\mathfrak{g}_{s_{1}, s_{2}}\right)=\sum_{s^{\prime}, s^{\prime \prime} \in S} \mathfrak{g}_{s^{\prime}, s^{\prime \prime}} V_{s, s_{1}}^{s^{\prime}} V_{s, s_{2}}^{s^{\prime \prime}} \quad \forall s, s_{1}, s_{2} \in S .
$$

\footnotetext{
${ }^{12}$ Via (7.1) a connection on $\hat{\Omega}^{1}(\mathcal{A}) \otimes_{L} \hat{\Omega}^{1}(\mathcal{A})$ defines a connection on $\hat{\Omega}^{1}(\mathcal{A}) \otimes_{\mathcal{A}} \hat{\Omega}^{1}(\mathcal{A})$ and vice versa. In Refs. [19] a left $\mathcal{A}$-module map $\sigma: \hat{\Omega}^{1}(\mathcal{A}) \otimes_{\mathcal{A}} \hat{\Omega}^{1}(\mathcal{A}) \rightarrow \hat{\Omega}^{1}(\mathcal{A}) \otimes_{\mathcal{A}} \hat{\Omega}^{1}(\mathcal{A})$, which generalizes the permutation map, has been used to extend a linear connection from $\hat{\Omega}^{1}(\mathcal{A})$ to $\hat{\Omega}^{1}(\mathcal{A}) \otimes_{\mathcal{A}} \hat{\Omega}^{1}(\mathcal{A})$ by $\nabla\left(\theta^{s} \otimes_{\mathcal{A}} \theta^{s^{\prime}}\right):=$ $\nabla \theta^{s} \otimes_{\mathcal{A}} \theta^{s^{\prime}}+\left(\sigma \otimes_{\mathcal{A}}\right.$ id $)\left(\theta^{s} \otimes_{\mathcal{A}} \nabla \theta^{s^{\prime}}\right)$. Though this is very different from our approach, the problems addressed in Refs. 19 can also be treated in our framework.
} 
In the special case where $\mathfrak{g}_{s, s^{\prime}} \in \mathcal{Z}(\mathcal{A})$, this can be written in matrix form as $\phi_{s}(\mathfrak{g})=V_{s}^{T} \mathfrak{g} V_{s}$.

A torsion-free linear connection which is compatible with a metric $\mathrm{g}$ is called a Levi-Civita connection of the respective metric. The conditions of vanishing torsion and compatibility with a given metric need not have any solution. If they do, it need not be unique [6].

Example 7.1. Let $(\hat{\Omega}(\mathcal{A}), \mathrm{d})$ be the bicovariant differential calculus on $G L_{p, q}(2)$ treated in section [5.2. A metric can then be expressed in the form (7.4) with respect to the basis of 1-forms given in (5.23). Using (5.27), the invariance condition (17.5) imposes the following conditions on the metric components which we assume to be symmetric: $\phi_{s}\left(\mathfrak{g}_{s^{\prime}, s^{\prime \prime}}\right)=\mathfrak{g}_{s^{\prime}, s^{\prime \prime}}$ if $s^{\prime}, s^{\prime \prime}=1,3,4, \phi_{s}\left(\mathfrak{g}_{2, s^{\prime}}\right)=r \mathfrak{g}_{2, s^{\prime}}$ if $s^{\prime}=1,3,4$, and $\phi_{s}\left(\mathfrak{g}_{2,2}\right)=r^{2} \mathfrak{g}_{2,2}$. Here the action of $\phi_{s}$ on $\mathcal{A}$ is determined by (5.26). In order to obtain a non-degenerate metric, some of its components must lie in $\mathcal{A} \backslash \mathcal{Z}(\mathcal{A})$. (17.7) determines corresponding compatible linear connections.

\section{Conclusions}

In section 4 we gave a recipe to construct (distinguished) differential calculi over an associative algebra $\mathcal{A}$ from subsets of automorphisms of $\mathcal{A}$. A differential calculus obtained in this way is actually just the differential calculus associated with the group lattice (Cayley digraph) $(G, S)$ according to Ref. [5. It is simply carried over to $\mathcal{A}$ via an action of $G$ by automorphisms of $\mathcal{A}$ (provided some technical requirements are fulfilled). Familiar differential calculi, like those on the quantum plane, turned out to be of this kind. We may conclude that they are in fact basically group lattice differential calculi! The Wess-Zumino calculus on the quantum plane [17] in this way corresponds to a $\mathbb{Z}^{2}$ lattice. Indeed, this relation can even be established in a more explicit way, see appendix A.

The correspondence with group lattices only becomes visible in a special coframe $\left\{\theta^{s} \mid s \in\right.$ $S$ \}. It should be noticed that the $\theta^{s}$ are not, in general, ordinary 1-forms. Either one has to extend (or rather restrict) the algebra $\mathcal{A}$ by appending further generators and relations, or one has to enlarge the bimodule of 1 -forms.

Geometric structures like linear connections and metrics are also easily carried over from the differential geometry of the associated group lattice. More precisely, this refers to expressions in terms of products of the $\theta^{s}$ with coefficients in the field $\mathbb{k}$. Clearly, the resulting geometry over $\mathcal{A}$ is richer since the coefficients lie more generally in $\mathcal{A}$.

Whenever an automorphism group of a noncommutative algebra $\mathcal{A}$ is given (see Refs. 15. 20 for further examples), following the recipe of section 4 distinguished differential calculi can be constructed and thus a natural basis for differential geometric structures on these algebras. Much of the formalism developed in Refs. 5, 6] for group lattices then generalizes to these algebras.

We should stress, however, that the construction of differential calculi in section 4 does not exhaust the possibilities admitting the structure (1.2), as demonstrated in particular in section 5.2 with an example of a bicovariant differential calculus on $G L_{p, q}(2)$. This led us to a generalization of the construction of section 4 involving twisted inner derivations.

Many examples of noncommutative field theories are constructed by deformation quan- 
tization of classical field theories (see [21], for example). Basically this amounts to replacing the classical product of functions by a Moyal $\star$-product, which results in a noncommutative algebra $\mathcal{A}$. On such algebras one can still establish an action of a (discrete) group. Suppose we start with a lattice gauge theory. One should then try to extend the action of the discrete translations (some $\mathbb{Z}^{n}$ ) to the deformed algebra (see Ref. [22, for example). Our work provides a basis for corresponding geometric models.

\section{A Realizations of some differential calculi}

Let $\mathcal{A}=\mathcal{A}_{1} \otimes \mathcal{A}_{2}$ with two associative algebras $\mathcal{A}_{1}, \mathcal{A}_{2}$ over a field $\mathbb{k}$, and $\left(\Omega_{1}\left(\mathcal{A}_{1}\right), \mathrm{d}_{1}\right)$ a differential calculus over $\mathcal{A}_{1}$. Defining $\mathrm{d}\left(\omega_{1} \otimes f_{2}\right):=\left(\mathrm{d} \omega_{1}\right) \otimes f_{2}$ for all $\omega_{1} \in \Omega_{1}$ and $f_{2} \in \mathcal{A}_{2}$, we obtain a differential calculus over $\mathcal{A}$. In the special case where $\mathcal{A}_{1}$ is commutative, the above tensor product yields an algebra isomorphism $\mathcal{A}_{2} \mapsto \mathcal{A}$. In this case, the above construction of a differential calculus carries a differential calculus over the commutative algebra $\mathcal{A}_{1}$ to a differential calculus over the (in general noncommutative) algebra $\mathcal{A}$, respectively $\mathcal{A}_{2}$ due to the isomorphism.

Example A.1. Let $u, v$ freely generate a commutative algebra and let $U, V$ be generators of a quantum plane, i.e. $U V=q V U$ where $q$ is a fixed parameter. Then

$$
x=u \otimes U, \quad y=v \otimes V
$$

also generate a quantum plane with $x y=q y x$. A differential calculus over the commutative algebra is defined by

$$
u \mathrm{~d} u=p q \mathrm{~d} u u, \quad v \mathrm{~d} u=p q \mathrm{~d} u v, \quad u \mathrm{~d} v=\mathrm{d} v u+(p q-1) \mathrm{d} u v, \quad v \mathrm{~d} v=p q \mathrm{~d} v v .
$$

In fact, introducing $w$ with $v=u w$, this becomes

$$
u \mathrm{~d} u=p q \mathrm{~d} u u, \quad w \mathrm{~d} u=\mathrm{d} u w, \quad u \mathrm{~d} w=\mathrm{d} w u, \quad w \mathrm{~d} w=p q \mathrm{~d} w w,
$$

which is the "lattice differential calculus" of Ref. 13. It is easily verified that the differential calculus induced on the tensor product of the two algebras satisfies the relations (4.41) in terms of the generators (A.1). In this way the differential calculus on the quantum plane originates from the lattice calculus over the commutative algebra generated by two variables, which may be realized as the algebra of functions on $\mathbb{Z}^{2}$. In this realization a field has the form $\psi(x, y)=\sum_{m, n} \psi_{m, n}(u, w) U^{m} V^{n}$ which can also be interpreted as a map from $\mathbb{Z}^{2}$ to the quantum plane algebra (generated by $U, V$ ). Field theory and differential geometry on the quantum plane are then essentially field theory and differential geometry on the lattice $\mathbb{Z}^{2}$.

Example A.2. Again, let $u, v$ freely generate a commutative algebra, but now we assume that $U, V$ satisfy the relations of the $h$-deformed plane: $[U, V]=h V^{2}$. This time we define the differential calculus over the commutative algebra by the relations

$$
[v, \mathrm{~d} v]=[v, \mathrm{~d} u]=[u, \mathrm{~d} v]=0, \quad[u, \mathrm{~d} u]=\left(h+h^{\prime}\right)(\mathrm{d} v u-\mathrm{d} u v) .
$$

Introducing $w$ with $u=v w$, we obtain the simpler structure

$$
[v, \mathrm{~d} v]=[v, \mathrm{~d} w]=[w, \mathrm{~d} v]=0, \quad[w, \mathrm{~d} w]=-\left(h+h^{\prime}\right) \mathrm{d} w .
$$


The differential calculus introduced on the tensor product now satisfies the relations (4.55) with $r=1$, where

$$
x=v \otimes U+u \otimes V, \quad y=v \otimes V .
$$

In this way the differential calculus on the $h$-deformed plane originates from the natural differential calculus over the algebra of functions on $\mathbb{R} \times \mathbb{Z}$. The conclusions are similar to those of the previous example.

It is interesting to see that prominent examples of differential calculi over noncommutative algebras can indeed be realized as a tensor product of a differential calculus over a commutative algebra with the noncommutative algebra itself. A lot of further examples can be constructed by using the differential calculi over commutative algebras presented in Refs. [5, 23] and arbitrary associative algebras.

\section{References}

[1] Madore J 1999 An Introduction to Noncommutative Differential Geometry and its Physical Applications (Cambridge: Cambridge University Press)

[2] Kunz E 1986 Kähler Differentials (Braunschweig: Vieweg);

Berndt R 2002 Kähler differentials and some applications in arithmetical geometry Hamburger Beiträge zur Mathematik, Heft 136

[3] Karoubi M 1999 Méthodes quantiques en topologie algébrique, preprint; 2001 Quantum methods in algebraic topology Contemp. Math. 279 177-193; 2000 Braiding of differential forms and homotopy types, C. R. Acad. Sci. Paris 331, Série I, 757-762;

Karoubi M and Alvarez M S 2001 Twisted Kähler differential forms, to appear in $J$. Pure Appl. Algebra

[4] Manin Yu I 1992 Notes on quantum groups and quantum de Rham complexes Theor. Math. Phys. 92 997-1019;

Dimakis A and Müller-Hoissen F 1992 Quantum mechanics on a lattice and qdeformations Phys. Lett. B 295 242-248

[5] Dimakis A and Müller-Hoissen F 2003 Differential geometry of group lattices J. Math. Phys. 44 1781-1821 math-ph/0207014

[6] Dimakis A and Müller-Hoissen F 2003 Riemannian geometry of bicovariant group lattices J. Math. Phys. 44 4220-4259 math-ph/0212054

[7] Dimakis A and Madore J 1996 Differential calculi and linear connections J. Math. Phys. $374647-4661$ q-alg/9601023

[8] Woronowicz S L 1989 Differential calculus on compact matrix pseudogroups (quantum groups) Commun. Math. Phys. 122 125-170

[9] Klimyk A U and Schmüdgen K 1997 Quantum Groups and Their Representations (Berlin: Springer) 
[10] Müller-Hoissen F 1992 Differential calculi on the quantum group $G L_{p, q}(2)$ J. Phys. A $251703-1734$

[11] Carow-Watamura U, Schlieker M, Watamura S and Weich W 1991 Bicovariant differential calculus on quantum groups $S U_{q}(N)$ and $S O_{q}(N)$ Commun. Math. Phys. 142 605-641;

Jurčo B 1991 Differential calculus on quantized simple Lie groups Lett. Math. Phys. 22 177-186;

Schmüdgen K and Schüler A 1995 Classification of bicovariant differential calculi on quantum groups of type A, B, C and D Commun. Math. Phys. 167 635-670;

Heckenberger I and Schmüdgen K 1998 Classification of bicovariant differential calculi on the quantum groups $S L_{q}(n+1)$ and $S p_{q}(2 n)$ J. reine angew. Math. 502 141-162

[12] Schmüdgen K 1998 On the construction of covariant differential calculi on quantum homogeneous spaces J. Geom. Phys. 30 23-47;

Hermisson U 1998 Construction of covariant differential calculi on quantum homogeneous spaces Lett. Math. Phys. 46 313-322

[13] Dimakis A, Müller-Hoissen F and Striker T 1993 Non-commutative differential calculus and lattice gauge theory J. Phys. A 26 1927-1949; 1993 From continuum to lattice theory via deformation of the differential calculus Phys. Lett. B 300 141-144

[14] Dimakis A and Müller-Hoissen F 1994 Discrete differential calculus: graphs, topologies, and gauge theory J. Math. Phys. 35 6703-6735

[15] Alev J and Chamarie M 1992 Dérivations et automorphismes de quelques algèbres quantiques Comm. Algebra 20 1787-1802

[16] Schirrmacher A 1991 The multiparametric deformation of $G L(n)$ and the covariant differential calculus on the quantum vector space Z. Phys. C 50 321-328

[17] Pusz W and Woronowicz S L 1989 Twisted second quantization Rep. Math. Phys. 27 231-257;

Wess J and Zumino B 1990 Covariant differential calculus on the quantum hyperplane Nucl. Phys. (Proc. Suppl.) B 18 302-312;

Brzeziński T, Dabrowski H and Rembieliński J 1992 On the quantum differential calculus and the quantum holomorphicity J. Math. Phys. 33 19-24;

Schmüdgen K and Schüler A 1993 Covariant differential calculi on quantum spaces and on quantum groups C. R. Acad. Scie. Paris 316 1155-1160

[18] Aghamohammadi A 1993 The 2-parametric extension of $h$ deformation of $G L(2)$, and the differential calculus on its quantum plane Mod. Phys. Lett. A 8 2607-2613 hep-th/9306071

[19] Mourad J 1995 Linear connections in non-commutative geometry Class. Quantum Grav. 21 965-974;

Dubois-Violette M, Madore J, Masson T and Mourad J 1995 Linear connections on the quantum plane Lett. Math. Phys. 35 351-358 hep-th/9410199; 
Georgelin Y, Masson T and Wallet J-C 1996 Linear connections on the two parameter quantum plane Rev. Math. Phys. 8 1055-1060 q-alg/9507032;

Aghamohammadi A, Khorrami M and Shariati A $1997 S L_{h}(2)$-symmetric torsionless connections Lett. Math. Phys. 40 95-99;

Cho S, Madore J and Park K S 1998 Noncommutative geometry of the h-deformed quantum plane J. Phys. A: Math. Gen. 31 2639-2654 q-alg/9709007

[20] Gómez-Torrecillas J and Kaoutit L EL 2002 The group of automorphisms of the coordinate ring of quantum symplectic space Contrib. to Algebra and Geometry 43 597-601

[21] Douglas M R and Nekrasov N A 2001 Noncommutative field theory Rev. Mod. Phys. 73 977-1029 hep-th/0106048

[22] Ambjørn J, Makeenko Y M, Nishimura J and Szabo R J 2000 Lattice gauge fields and discrete noncommutative Yang-Mills theory JHEP 0005023 hep-th/0004147;

Szabo R J 2001 Discrete noncommutative gauge theory 2001 Mod. Phys. Lett. A 16 367-386 hep-th/0101216

[23] Baehr H, Dimakis A and Müller-Hoissen F 1995 Differential calculi on commutative algebras J. Phys. A 28 3197-3222 hep-th/9412069 